\title{
Undulating changes in human plasma proteome across lifespan are linked to disease
}

\author{
Benoit Lehallier ${ }^{1,2,3^{*}}$, David Gate ${ }^{1,2,3,4}$, Nicholas Schaum ${ }^{5}$, Tibor Nanasi ${ }^{1,2,3,6}$, Song Eun Lee ${ }^{1,2,3,4}$, \\ Hanadie Yousef ${ }^{1,2,3,4}$, Patricia Moran Losada ${ }^{1,2,3}$, Daniela Berdnik ${ }^{1,2,3,4}$, Andreas Keller ${ }^{7}$, \\ Joe Verghese ${ }^{8,9}$, Sanish Sathyan ${ }^{8,9}$, Claudio Franceschi ${ }^{10,11}$, Sofiya Milman ${ }^{8,12}$, Nir Barzilai ${ }^{8,12}$, \\ Tony Wyss-Coray ${ }^{1,2,3,4 *}$ \\ ${ }^{1}$ Department of Neurology and Neurological Sciences, Stanford University, Stanford, CA, USA \\ ${ }^{2} \mathrm{Wu}$ Tsai Neurosciences Institute, Stanford University, Stanford, CA, USA \\ ${ }^{3}$ Paul F. Glenn Center for the Biology of Aging, Stanford University, Stanford, CA, USA \\ ${ }^{4}$ Department of Veterans Affairs, VA Palo Alto Health Care System, Palo Alto, CA, USA \\ ${ }^{5}$ Institute for Stem Cell Biology and Regenerative Medicine, Stanford University, Stanford, CA, USA \\ ${ }^{6}$ Institute of Cognitive Neuroscience and Psychology, Hungarian Academy of Sciences Research Centre for Natural \\ Sciences, Budapest, Hungary \\ ${ }^{7}$ Clinical Bioinformatics, Saarland University, Saarbrücken, Germany. \\ ${ }^{8}$ Institute for Aging Research, Department of Medicine, Albert Einstein College of Medicine, Bronx, NY, USA \\ ${ }^{9}$ Department of Neurology, Albert Einstein College of Medicine, Bronx, NY, USA \\ ${ }^{10}$ University of Bologna, Bologna, Italy \\ ${ }^{11}$ National Research Lobachevsky State University of Nizhny Novgorod, Russia \\ ${ }^{12}$ Department of Genetics, Albert Einstein College of Medicine, Bronx, NY, USA \\ *Correspondence: twc@stanford.edu (T.W-C.) and lehallib@stanford.edu (B.L.)
}

Aging is the predominant risk factor for numerous chronic diseases that limit healthspan. Mechanisms of aging are thus increasingly recognized as therapeutic targets. Blood from young mice reverses aspects of aging and disease across multiple tissues, pointing to the intriguing possibility that age-related molecular changes in blood can provide novel insight into disease biology. We measured 2,925 plasma proteins from 4,331 young adults to nonagenarians and developed a novel bioinformatics approach which uncovered profound non-linear alterations in the human plasma proteome with age. Waves of changes in the proteome in the fourth, seventh, and eighth decades of life reflected distinct biological pathways, and revealed differential associations with the genome and proteome of age-related diseases and phenotypic traits. This new approach to the study of aging led to the identification of unexpected signatures and pathways of aging and disease and offers potential pathways for aging interventions.

Aging underlies progressive changes in organ functions and is the primary risk factor for a large number of human disease ${ }^{1}$. A deeper understanding of aging is thus likely to provide insight into the underlying mechanisms of disease and facilitate the development of novel therapeutics that target the aging process more generally. A growing number of investigators have applied genomic, transcriptomic and proteomic assays (collectively referred to as omics) to studies of aging from model organisms to humans ${ }^{2}$. Human genetic studies have uncovered relatively few modifiers of aging, yet other omics modalities, which measure more dynamic gene modifications or products, have provided valuable insight. For example, the transcriptome varies greatly during aging across tissues and organisms ${ }^{3}$, pointing to evolutionarily conserved, fundamental roles of developmental and inflammatory pathways ${ }^{4}$. Protein composition of cells, body fluids, and tissues change similarly with age and provide insight into complex biological processes since proteins 
are often direct regulators of cellular pathways. In particular, blood, which contains proteins from nearly every cell and tissue, has been extensively analyzed to discover biomarkers and gain insight into disease biology. Accordingly, organismal aging results in proteomic changes in blood that reflect aspects of aging of different cell types and tissues.

Perhaps the strongest evidence that blood can be used to study aging comes from experiments employing heterochronic parabiosis, a surgically induced state in which the circulatory systems of young and old mice are joined. These studies show that multiple tissues, including muscle, liver, heart, pancreas, kidney, bone, and brain can be rejuvenated in old mice ${ }^{5-}$ 13. Plasma (the soluble fraction of blood) from old mice is sufficient to accelerate aspects of brain aging following repeated infusion into young mice ${ }^{12}$ and young plasma can slow and reverse memory impairment and other aspects of brain aging ${ }^{13,14}$. Altogether, these studies support the notion that the plasma proteome harbors key regulators of aging. Identifying such protein signatures may help understand mechanisms of organismal aging. However, blood proteomic changes with age have not been thoroughly exploited and require novel tools to derive insight into the biology of aging. Here, we carried out a deep proteomic analysis of plasma from young adults to nonagenarians and applied newly developed data analysis tools. We discovered distinct changes in protein expression across stages of lifespan and link these changes to biological pathways and disease.

\section{Results}

\section{Linear modeling links the plasma proteome to functional aging and identifies a conserved aging signature.}

We analyzed plasma isolated from ethylenediaminetetraacetic acid (EDTA)-treated blood acquired via venipuncture from 4,331 healthy individuals aged 18-95 years from the Interval and LonGenity cohorts (Fig. 1a, Supplementary Fig. 1). Currently, one of the most advanced tools for the measurement of plasma proteins are single-stranded oligonucleotides known as aptamers ${ }^{15,16}$, which bind their target with high affinity and specificity. To generate a proteomic dataset of human lifespan, we used the SomaScan aptamer technology, capable of quantifying thousands of proteins involved in intercellular signaling, extracellular proteolysis, and metabolism (Supplementary Tables 1 and 2). Reproducibility analysis based on technical replicates showed an overall low median coefficient of variation (CV) of $\sim 5 \%$ within runs and $10 \%$ between runs ${ }^{17}$ (Supplementary Table 3). The Interval and LonGenity proteomics datasets analyzed in this study can be interrogated by an interactive web interface (https://twcstanford.shinyapps.io/aging plasma proteome/).

Since females have longer average lifespans than males independent of socio-economic status $^{18}$, we sought to determine whether sex and aging proteomes are interconnected (Fig $1 \mathrm{~b}$ 1d). Proteins most strongly changed with sex included well-known follicle stimulating hormone (CGA FSHB), human chorionic gonadotropin (CGA CGB), and prostate-specific antigen (KLK3). With age, the most prominent overall changes with respect to fold change and statistical significance included sclerostin (SOST), ADP Ribosylation Factor Interacting Protein 2 (ARFIP2), and growth differentiation factor 15 (GDF15), in addition to several sex proteins such as CGA FSHB. The proteins most strongly associated with age also changed significantly with sex, (Fig. 1d); 895 proteins out of the 1,379 proteins altered with age $(q<0.05)$ were significantly different between males and females $(q<0.05$, Supplementary Table 4$)$. These results are aligned with a growing number of studies demonstrating that males and females age differently ${ }^{19}$. To determine whether these findings are representative of the general population, we compared changes identified in this study with findings from 4 small independent cohorts ( $n=171$, age range 21-107y, Supplementary Fig. 1d) and with findings from an independent study ${ }^{20}$. Even though these independent studies used an older version of the SomaScan assay measuring only a subset of 
the current proteins (1,305 proteins; Supplementary Table 2), we observed high consistency of the aging and sex proteomes across cohorts (Supplementary Fig. 2).

To establish the biological relevance of these changes, we queried GO, KEGG and Reactome databases and measured enrichment of proteins in pathways using a "Sliding Enrichment Pathway Analysis (SEPA)" (Supplementary Tables 5 and 6, see Methods). The heatmaps produced by SEPA firstly illustrate the relationship between the top 100 proteins and the biological pathways they represent; secondly, the heatmaps emphasize how a restricted list of top aging proteins reveal biological pathways which would have escaped common pathway mining modalities. SEPA indicated that incremental lists of proteins are needed to determine biological functions of sex proteins, and pointed to expected differences in hormonal metabolism and activity (Fig. 1e, Supplementary Table 6). Conversely, an extensive list of aging proteins identified enrichment for blood-related pathways such as heparin and glycosaminoglycan binding, as recently reported ${ }^{20}$.

To determine whether the plasma proteome can predict chronological age and serve as a "proteomic clock," we used 2,858 randomly selected subjects to fine-tune a predictive model that was tested on the remaining 1,473 subjects (Fig. 1f). We identified a sex-independent plasma proteomic clock consisting of 373 proteins (Supplementary Table 7). This clock was highly accurate in predicting chronological age in the discovery, validation and 4 small independent cohorts ( $r=0.93-0.97$, Fig. $1 \mathrm{~g}$ and Supplementary Fig. 3a-b). Remarkably, subjects that were predicted younger than their chronologic age based on their plasma proteome performed better on cognitive and physical tests (Fig. $1 \mathrm{~h}$ and Supplementary Table 8 ). While a reduced model comprising only 9 proteins predicted age with good accuracy (Supplementary Fig.3c and Supplementary Table 7), a combination of different sets of proteins may be required to model changes in a large set of clinical and functional parameters (Supplementary Fig.3d).

Since most biological pathways that change with age are evolutionarily conserved from yeast to mammals ${ }^{21}$, we tried to identify possible aging proteins conserved between mice and humans. Thus, we analyzed mouse plasma ( $n=110$, aged 1 month to 30 months) using the SomaScan assay, which reliably measure hundreds of proteins in samples from non-human species $^{22}$ and has proven useful in some applications in recent mouse studies ${ }^{9,23}$ (Fig. 1i, Supplementary Table 9). In mice, 172 proteins changed with age (out of 1,305 measured, Supplementary Table 10, $\mathrm{q}<0.05$ ) and 46 proteins overlapped with human aging proteins (Fig. 1j). Remarkably, many of these proteins were modulated by heterochronic parabiosis: young mice exposed to old plasma (young-heterochronic mice) showed a relatively older plasma signature while aged mice exposed to young plasma (old-heterochronic mice) showed a younger signature (Fig. 1k). Altogether, standard linear modeling of the plasma proteome during human lifespan revealed established aging pathways, possibly indicating accelerated and decelerated aging in humans and mice. Intriguingly, changes to the conserved aging proteins did not occur simultaneously (Fig. 11). Thus, the chronology of aging in the plasma proteome requires further investigation.

\section{Clustering individual protein trajectories reveals undulation of the aging plasma proteome.}

While the above standard linear modeling showed prominent overall changes in plasma protein composition, we were struck by the undulating behavior of the 46 conserved proteins (Fig. 1I) and more globally by the 2,925 plasma proteins as a group when they were visualized as $\mathrm{Z}$ scored changes across lifespan (Fig. 2a-b). These undulating patterns were detected in independent human cohorts and in mice (Supplementary Fig. 4), suggesting they are robust and conserved across species.

To reduce complexity of these changes, we grouped proteins with similar trajectories using unsupervised hierarchical clustering (Fig. 2c) and identified 8 clusters of protein trajectories changing with age, which ranged in size from 8 to 1,415 proteins (Supplementary Table 11). In addition to linear trajectories (clusters 1, 4 and 5), several non-linear trajectories including 
stepwise, logarithmic and exponential changes were also evident (clusters 2, 3, 6, 7 and 8) (Fig. 2d). Remarkably, these cluster trajectories were similarly detectable in independent cohorts (Supplementary Fig. 5). Out of the 8 clusters analyzed, 6 were enriched for specific biological pathways ( $q<0.05$; Supplementary Fig. 6, Supplementary Table 12), suggesting distinct, yet orchestrated changes in biological processes during the human lifespan. For example, proteins present in blood microparticles consistently decreased with age (cluster 5); other blood-related pathways such as heparin and glycosaminoglycan binding increased in a two-step manner (cluster 4); while levels of proteins involved in axon guidance and EPH-ephrin signaling remain constant until age 60 before they rise exponentially (cluster 6) (Fig. 2d). Altogether, we find that the majority of changes in the plasma proteome during lifespan occur in a non-linear manner.

\section{Quantification of proteomic changes across human lifespan uncovers waves of aging proteins.}

To gain a quantitative understanding of the proteomic changes occurring throughout human lifespan, we developed the software tool Differential Expression - Sliding Window ANalysis (DE-SWAN) (Fig. 3a). This algorithm analyzes levels of plasma proteins within a window of 20 years and compares two groups of individuals in parcels of 10 years (e.g. 35-45 compared with 45-55). We used a window of 20 years to detect age related-changes and slid the window in increments of 1 year from young to old. By this method, we aimed to detect changes at particular stages of lifespan and determine the sequential effects of aging on the plasma proteome (while also controlling for the effect of confounding factors). This approach identified hundreds of plasma proteins changing in waves throughout age (Fig. 3b). Summing the number of differentially expressed proteins at each age uncovered 3 crests at ages 34, 60, and 78 (Fig. 3c, Supplementary Fig. 7a and Supplementary Table 13). These crests disappeared when the ages of individuals were permutated (Supplementary Fig. 7b), but were still detectable when using different statistical models (e.g. smaller/larger sliding windows) (Supplementary Fig. 7c, and 8, Supplementary Table 13), indicating robustness of these age-related waves of plasma proteins.

The 3 age-related crests were comprised of different proteins (Fig. 3d, Supplementary Table 14). Few proteins, such as GDF15, were among the top 10 differentially expressed proteins in each crest, consistent with its strong increase across lifespan (Fig. 3a). Other proteins, like chordin-like protein 1 (CHRDL1) or pleiotrophin (PTN), were significantly changed only at the last two crests, reflecting their exponential increase with age. The overlap between the sets of proteins changing at age 34,60 and 78 was statistically significant $(p<0.05)$ but limited (Fig. $3 e)$ and most of the proteins changing in old age were not identified by linear modeling (Fig. 3f). This prompted us to use SEPA to determine whether these waves reflected distinct biological processes. Strikingly, we observed a prominent shift in multiple biological pathways with aging (Fig. $3 g$ ). At young age (34 years), we observed a downregulation of proteins involved in structural pathways such as the extracellular matrix (ECM). These changes were reversed in middle and old ages (60 and 78 years, respectively). At age 60 , we found a predominant role of hormonal activity, binding functions and blood pathways. At age 78, key processes still included blood pathways but also bone morphogenetic protein signaling, which is involved in numerous cellular functions, including inflammation ${ }^{24}$. Pathways changing with age according to linear modeling (Fig. 1g) overlapped most strongly with the crests at age 34 and 60 (Fig. 3f), indicating the dramatic changes in protein expression occurring in the elderly might be masked in linear modelling by more subtle changes at earlier ages. Altogether, these results suggest that aging is a dynamic, non-linear process characterized by waves of changes in plasma proteins that are reflective of a complex shift in the activity of biological processes.

\section{Proteins linked to age-related diseases are enriched in distinct waves of aging.}

The plasma proteome is sensitive to the physiological state of an individual but is also genetically determined ${ }^{25}$. To deconvolute this complexity between genome, proteome, and 
physiology, we asked whether the top plasma aging proteins change their levels due to genetic polymorphisms ( $p Q T L s$ ) or whether they are among the top predictors of disease, or phenotypic traits. More specifically, we sought to determine whether proteins that comprised the 3 waves of aging were uniquely linked to the genome or proteome of age-related diseases and traits (Fig. 4a). To this end, we used the ranked lists of the top proteins identified by DE-SWAN at each of the three crests (Fig. 3c and Supplementary Table 14) and summed the number of proteins linked to the genome and proteome of specific diseases and traits separately for each wave (i.e. the cumulative sum) (Fig. 4b-i and Supplementary Fig. 9). First, we mined the genomic atlas of the human plasma proteome ${ }^{25}$ (Fig. 4b) and discovered that the aging proteome is also genetically determined (Fig.4c and Supplementary Fig. 9). However, the rank of the proteins determined by trans-association appeared more random with aging (Fig. 4c), suggesting that other sources are driving the aging plasma proteome with age. We then tested whether the waves of aging proteins were differentially linked with changes in cognitive and physical functions identified in Fig. $1 \mathrm{~h}$. Interestingly, the proteome associated with these traits overlapped with the proteome defining middle and old ages, i.e. when these functions decline the most (Fig. 4d and Fig. 4e). Finally, we used public datasets and summary statistics from SomaScan proteomic studies focusing on agerelated diseases including Alzheimer's Disease $(A D)^{26}$, Down Syndrome $(D S)^{27}$ and cardiovascular disease (CVD) ${ }^{28}$. A proteomic study predicting body mass index (BMI $)^{29}$ based on the plasma proteome was used as a control since weight gain varies widely with age according to data from the US National Health and Nutrition Examination Study ${ }^{30}$ (Supplementary Fig. 10). As expected, the proteome linked to BMI was not selectively enriched for proteins defining waves of aging (Fig. 4f). CVD-associated proteins were strongly enriched in waves of proteins defining middle and old age compared to young age (Fig. $4 \mathrm{~g}$ ). This enrichment corresponded to an increased incidence of CVD after 55 years ${ }^{31}$. Finally, AD- and DS-associated proteins overlapped with the top proteins defining middle age and old age but not with proteins in the young wave of aging (Fig. 4h-i). The fact that the proteome defining these two diseases also changed in old individuals of a separate disease-free cohort supports the notion of accelerated aging in DS and $A D^{32,33}$. Altogether, these results show that the waves of proteomic aging are differentially linked to the genomic and proteomic traits of various diseases.

\section{Discussion}

Our analysis of 2,925 proteins in plasma from healthy humans reveals complex, non-linear changes during human lifespan. Although modeling protein trajectories is necessary to truly appreciate these undulating changes, a standard linear analysis provides key information about the aging plasma proteome.

It is well known that men and women age differently ${ }^{19}$, but we were surprised to find that $2 / 3$ of the proteins changing with age are also changing with sex (895 proteins out of the 1,379 proteins changing with age). These results strongly support the National Institutes of Health (NIH) policy on the inclusion of women in clinical research and the inclusion of sex as a biological variable in scientific experiments. Nevertheless, a unique proteomic clock can be used to predict age in men and women and deviations from this plasma proteomic clock are correlated with changes in clinical and functional parameters (Fig 1g-h). The panel of 373 proteins or a subset can be used to assess the relative health of an individual and to measure healthspan, analogous to epigenetic clocks based on DNA methylation patterns ${ }^{34}$. More large-scale plasma proteomic studies will be required to establish the validity and utility of this clock and to test if specific subsets of proteins may be more appropriate to reflect particular clinical and functional parameters.

Blood is a sensitive marker of functional aging but also plays an active role in aging. Numerous studies have shown that soluble factors from young mouse blood reverse aspects of aging and disease across multiple tissues ${ }^{5-13,35}$. Here, we describe a 46-protein aging signature that is conserved in humans and mice, containing several known aging proteins such GDF15 ${ }^{36}$ 
and IGF1/INSR ${ }^{37}$ but also less investigated ones (Fig 1I). This conserved signature will allow deeper investigation of translational aging interventions in mice, such as heterochronic parabiosis, which partially reverses age-related changes of these proteins (Fig $1 \mathrm{k}$ ).

By deep mining the aging plasma proteome, we identify undulating changes during the human lifespan. These changes are the result of clusters of proteins moving in distinct patterns, culminating in the emergence of 3 waves of aging. Somewhat unexpectedly, we find that these clusters are often part of shared biological pathways, most notably in cellular signaling functions (Fig. 2). In addition, we identify and provide biological relevance for the 3 main waves of aging proteins (Fig. 3). These waves are characterized by key biological pathways with little overlap, demonstrating changes to chronological aging in the plasma proteome. In comparison, linear modeling fails to identify changes occurring late in the $8^{\text {th }}$ decade of life (Fig. $3 \mathrm{~g}$ ). We conclude that linear modeling of aging based on "omics" data does not capture the complexity of biological aging across organismal lifespan. Thus, DE-SWAN will be invaluable for analyzing longitudinal or cross-sectional datasets with non-linear, quantifiable changes, and for integrating non-linear changes in the analysis of high dimensional "omics" datasets of a quantitative trait.

Sources of variation of the plasma proteome can be diverse and a majority of the plasma proteome is under substantial genetic control ${ }^{25,38}$. Intriguingly, we observed that the relative importance of trans-associations decreased with aging (Fig. 4c). This led us to investigate other sources of variance with a focus on disease-associated proteomes and traits. We found that proteins comprising the middle and old age waves differentially overlapped with proteins associated with cognitive and physical impairments. Moreover, plasma proteins that changed most prominently with age also discriminated patients from age-matched controls in age-related diseases including AD, DS and CVD (Fig. 4g-i). This suggests that the characteristic plasma proteins of aging are amplified in age-related diseases. Using an AD- and DS-free cohort, we provide evidence to support the concept of accelerated aging for these two diseases ${ }^{32,33}$. Further investigation of these proteins is warranted to determine whether these associations indicate aging biomarkers and/or are causal proteins of disease. Nonetheless, these results suggest that variance within the aging plasma proteome slowly transitions from hard coding factors (i.e. genomic) to soft coding factors (e.g. diseases, environmental factors and resulting changes in cognitive and physiological functions).

The undulating nature of the aging plasma proteome and its interactions with diseases ought to be considered when developing proteomic signatures for diagnostic purposes. Indeed, disease proteomes overlap significantly with the waves of aging proteins (Supplementary Table 15). Taking into account the heterogeneous and non-linear changes of the plasma proteome during the entire lifespan can likely improve the sensitivity and specificity of prognostics and diagnostics tests. Moreover, these results are pertinent when considering the use of blood or blood products to treat aging and age-related diseases ${ }^{39}$. Specifically, identifying proteins within plasma that promote or antagonize aging at different stages of life could lead to more targeted therapeutics, as well as preventative therapies. Such reliable tests and treatments are still urgently needed for numerous diseases and, in the future, we hope to describe plasma proteome changes that predict subjects transitioning to disease. Of particular interest are studies of AD for which blood-based biomarkers are unavailable and clinical symptoms are believed to occur up to two decades after disease onset.

URLs.

https://twc-stanford.shinyapps.io/aging plasma proteome/

\section{Online content}


Any methods, additional references, Nature Research reporting summaries, source data, statements of data availability and associated accession codes are available at https://doi.org/xxxxyyyy.

\section{Acknowledgements}

We thank the members of the Wyss-Coray laboratory for feedback and support. We thank clinical staff for human blood-plasma collection/coordination. We thank Adam Butterworth for his help to get access to the Interval proteomics data.

The AddNeuroMed data are from a public-private partnership supported by EFPIA companies and European Union of the Sixth Framework program priority FP6-2004-LIFESCIHEALTH-5. Clinical leads responsible for data collection are Iwona Kłoszewska (Lodz), Simon Lovestone (London), Patrizia Mecocci (Perugia), Hilkka Soininen (Kuopio), Magda Tsolaki (Thessaloniki), and Bruno Vellas (Toulouse) and imaging leads Andy Simmons (London), Larsw Olad Wahlund (Stockholm) and Christian Spenger (Zurich) and bioinformatics leads are Richard Dobson (London) and Stephen Newhouse (London).

This work was supported by a National Institutes of Health National Institute on Aging (NIA) F32 1F32AG055255 01A1 (D.G.), the Cure Alzheimer's Fund (T.W-C.), the NOMIS Foundation (T.W-C.), the Stanford Brain Rejuvenation Project (an initiative of the Stanford Wu Tsai Neurosciences Institute), The Paul F. Glenn Center for Aging Research (T.W-C.), NIA R01 AG045034; DP1 AG053015 (T.W-C.) and the NIA funded Stanford Alzheimer's Disease Research Center P50AG047366, NIA K23AG051148 (S.M.), R01AG061155 (S.M.), American Federation for Aging Research (S.M.), R01AG044829 (J.V. and N.B), NIA R01AG057909 (N.B.), the Nathan Shock Center of Excellence for the Basic Biology of Aging P30AG038072 (N. B.), the Glenn Center for the Biology of Human Aging (N.B.).

\section{Author Contributions}

B.L. and T.W-C. planned the study, D.B., C.F., S.M., J.V., S.S. and N.B. provided human plasma samples, N. S., S.E.L and H.Y performed the mouse experiments, B.L analyzed the data with contributions from T.N. and A.K., P.M.L. developed the shiny app, B.L., D.G. and T.W-C. wrote the manuscript, A.K, C.F, S.M, J.V., S.S., N.B. and T.W-C. supervised the study, all authors edited and reviewed the manuscript.

\section{Competing Interests}

The authors declare no competing financial interests.

\section{Additional Information}

Supplementary information is available for this paper at https://doi.org/xxxxyyyt.

Reprints and permissions information is available at www.nature.com/reprints. Correspondence and requests for materials should be addressed to T.W-C or B.L.

Publisher's note: Springer Nature remains neutral with regard to jurisdictional claims in published maps and institutional affiliations. 


\section{Methods}

\section{Plasma proteomics measurements}

The SomaScan platform was used to quantify relative protein levels. This platform was established to identify biomarker signatures of diseases and conditions, including cardiovascular risk $^{28}$, cancer $^{40}$ and neurodegenerative disease ${ }^{26}$. The SomaScan platform is based on modified single-stranded DNA aptamers (SOMAmer ${ }^{\circledR}$ reagents) binding to specific protein targets. Assay details have been previously described ${ }^{17}$. Different versions of the SomaScan assay were used in the LonGenity, INTERVAL and the 4 independent human cohorts. These versions contained $5,284,4,034$, and 1,305 aptamers, respectively.

Out of the 4,034 aptamers measured in the INTERVAL cohort, 3,283 were contained in the publicly available dataset (https://ega-archive.org/studies/EGAS00001002555). Our study focuses on 2,925 aptamers with identical Seqld and SeqldVersion in both INTERVAL and LonGenity cohorts (Supplementary Table1). Out of the 2,925 aptamers, 888 were measured in the 4 independent cohorts and in mice (Supplementary Table 2).

\section{Human cohort characteristics}

INTERVAL cohort

Participants in the INTERVAL randomized controlled trial were recruited with the active collaboration of the National Health Service Blood and Transplant England (www.nhsbt.nhs.uk), which has supported field work and other elements of the trial. DNA extraction and genotyping was co-funded by the National Institute for Health Research (NIHR), the NIHR BioResource (http://bioresource.nihr.ac.uk/) and the NIHR Cambridge Biomedical Research Centre at the Cambridge University Hospitals NHS Foundation Trust. The INTERVAL study was funded by NHSBT (11-01-GEN). The academic coordinating center for INTERVAL was supported by core funding from: NIHR Blood and Transplant Research Unit in Donor Health and Genomics (NIHR BTRU-2014-10024), UK Medical Research Council (MR/L003120/1), British Heart Foundation (RG/13/13/30194) and the NIHR Cambridge Biomedical Research Centre at the Cambridge University Hospitals NHS Foundation Trust. Proteomic assays were funded by the academic coordinating center for INTERVAL and MRL, Merck \& Co., Inc. A complete list of the investigators and contributors to the INTERVAL trial has been previously reported ${ }^{41}$. The academic coordinating center would like to thank blood donor center staff and blood donors for participating in the INTERVAL trial.

Proteomics measurements from 3,301 human plasma samples (1,685 males and 1,616 females) from 2 different subcohorts were used for this study. Age ranged from 18 to 76 years with a median age of $45\left(1^{\text {st }}\right.$ Quartile $=31,3^{\text {rd }}$ Quartile $\left.=55\right)$. Sample selection, processing and preparation were detailed previously ${ }^{25}$.

LonGenity cohort

LonGenity is an ongoing longitudinal study initiated in 2008, designed to identify biological factors that contribute to healthy aging ${ }^{42}$. The LonGenity study enrolls older adults of Ashkenazi Jewish descent, age 65-94 years at baseline. Approximately $50 \%$ of the cohort consists of offspring of parents with exceptional longevity (OPEL), defined by having at least one parent that survived to 95 years of age. The other half of the cohort includes offspring of parents with usual survival (OPEL), who do not have a parental history of exceptional longevity. Proteomics measurements from 1,030 human plasma samples (457 males and 573 females) collected at baseline in LonGenity participants were used for this study. Age ranged from 61 to 95 years with a median 
age of $74\left(1^{\text {st }}\right.$ Quartile $=69,3^{\text {rd }}$ Quartile=80). LonGenity subjects are thoroughly characterized demographically and phenotypically at annual visits that include collection of medical history and physical and neurocognitive assessments. Sixty-eight subjects without clinical and functional data were excluded from the analysis. The LonGenity study was approved by the Institutional Review Board (IRB) at the Albert Einstein College of Medicine.

Additional 4 small independent cohorts

One hundred seventy-one human plasma samples (84 males and 87 females) were obtained from 4 different cohorts (VASeattle, PRIN06, PRIN09, GEHA). Age ranged from 21 to 107 years with a median of 70 years $\left(1^{\text {st }}\right.$ Quartile $=58,3^{\text {rd }}$ Quartile $\left.=89\right)$. Written informed consent was obtained for each subject. The IRB has determined that our research does not meet the definition of human subject research per Stanford's Human Research Protection Program policy and no IRB approval was required for this study.

For these cohorts, all samples were stored at $-80^{\circ} \mathrm{C}$ and $150 \mu \mathrm{l}$ aliquots of plasma were sent on dry ice to SomaLogic Inc. (Boulder, Colorado, US). Plasma samples were analyzed in three different batches, 24 samples in 2015, 70 in 2016 and 77 in 2017. In addition to these 171 plasma samples, 12 additional aliquots from 4 of these samples were measured in the different batches to estimate intra- and inter-assay variability (Supplementary Table 3). Data for 1,305 SOMAmer probes were obtained. No sample or probe data were excluded. HybNorm. plateScale.medNorm files provided by SomaLogic Inc. were bridged to data from the $1^{\text {st }}$ batch of samples using calibrators.

\section{Normalization of INTERVAL and LonGenity datasets}

Relative Fluorescent Units (RFU) of each plasma protein were log10-transformed. We normalized the levels of each protein within each subcohort based on the average of the subjects in the 6070 years range. Supplementary Fig. 11 shows representative normalization examples. Note that this normalization is needed when fitting aging trajectories (Fig. 2) but does not affect the results when "subcohort" is included as covariate in the modeling. The data from the 4 small independents cohorts were log10 transformed and bridged together using SomaLogic procedure based on calibrators. However, the number of samples in the 60-70 years range was too small to reliably bridge this data to the INTERVAL and LonGenity cohorts.

\section{Linear changes in the aging plasma proteome}

To determine the effect of age and sex at the protein level, we used the following linear model:

Protein level $\sim \alpha+\beta 1$ Age $+\beta 2$ Sex $+\beta 3$ Subcohort $+\varepsilon$

Type II sum of squares (SS) were calculated using the Anova function of the $R$ car package ${ }^{43}$. This SS type tests for each main effect after the other main effects. Q-values were estimated using Benjamini-Hochberg approach ${ }^{44}$. It should be noted that the age range differs between cohorts. If the adjustment for cohort effect decreases the number of false positives, it could also alter the true positive rate. In the 4 small independent cohorts, the "Subcohort" covariate also accounts for batch effect since samples from different cohorts were measured in different batches (except for PRIN06 and GEHA that were measured together).

To determine the relative proportion of variance explained by age and sex, we calculated the partial Eta2 as follows:

$$
\text { Partial Eta2 }=\frac{\mathrm{SS}_{\text {effect }}}{\left(\mathrm{SS}_{\text {effect }}+\mathrm{SS}_{\text {error }}\right)}
$$




\section{Validation of the aging proteins}

To provide confidence in the reproducibility of the protein assays, we compared our findings with the associations with age reported by Tanaka et al. ${ }^{20}$. To this end, we merged our results with those from Tanaka et al. using "Somald". Of note, Tanaka et al. used the same version of the SomaScan platform that we used for the 4 small independent cohorts (1,305 proteins).

\section{Sliding Enrichment Pathway Analysis (SEPA)}

To determine the biological meaning of group of plasma proteins, we ranked the top 100 proteins based on the product of -log10(p-values) and beta age and queried three of the most comprehensive biological annotation and pathway databases: Gene ontology - $\mathrm{GO}^{45}$, Kyoto Encyclopedia of Genes and Genomes - KEGG ${ }^{46}$ and Reactome ${ }^{47}$. Using these databases, we tested enrichment for pathways in the top 10 to top 100 proteins in increments of 1 protein. The 2,925 proteins measured in this study cover $90 \%$ of the human GOs, reactome and KEGG terms containing more than 8 genes (Supplementary Fig. 12).

To analyze each incremental list of proteins, we used the R TopGO package ${ }^{48}$ for $G O$ analysis and the $\mathrm{R}$ clusterprofiler package ${ }^{49}$ for KEGG and Reactome analyses. As input of SEPA, we used Gene Symbols provided by SomaLogic Inc. (Supplementary Table 1). The 2,925 proteins measured by SomaScan served as the background set of proteins against which to test for overrepresentation. Since several individual proteins (33 out of 2,925) were mapped to multiple Gene Symbols, we kept only the $1^{\text {st }}$ Gene Symbol provided by Somalogic to prevent false positive enrichment. For KEGG and Reactome analysis, clusterprofiler requires EntrezID as input. Therefore, we mapped Gene Symbols to EntrezID using the org.Hs.eg.db package ${ }^{50}$. Again, to avoid false positive enrichment, only the $1^{\text {st }}$ EntrezID was used when Gene Symbols were mapped to multiple EntrezID. Q-values were estimated using Benjamini-Hochberg approach ${ }^{44}$ for the different databases taken separately. For GO analysis, q-values were calculated for the three GOs classes (molecular function, cellular component, biological process) independently. To identify the most biologically meaningful terms and pathways, we reported only those with 20-500 proteins measured by the SomaScan assay. In addition, we focused on pathways consistently highly significant ( $q<0.05$ for at least 20 different incremental list of proteins) and kept the top ten pathways per condition (e.g. for each wave of aging proteins). Ranking was performed based on the minimum fdr across the incremental lists of proteins. SEPA can be viewed as an extension of the GSEA approach ${ }^{51}$, with more control for true and false positives.

\section{Validation of the aging signature in mice}

Male and virgin female C57BL/6JN mice were shipped from the National Institute on Aging colony at Charles River (housed at 67-73 ${ }^{\circ} \mathrm{F}$ ) to the Veterinary Medical Unit (VMU; housed at 68$\left.76{ }^{\circ} \mathrm{F}\right)$ ) at the VA Palo Alto (VA). At both locations, mice were housed on a 12-h light/dark cycle and provided food and water ad libitum. The diet at Charles River was NIH-31, and Teklad 2918 at the VA VMU. Littermates were not recorded or tracked. Mice 18-months-old and younger were housed at the VA VMU for no longer than 2 weeks before euthanasia, and mice older than 18months were housed at the VA VMU until they reached the experimental age. After anaesthetization with $2.5 \% \mathrm{v} / \mathrm{v}$ Avertin, blood was drawn via cardiac puncture. All animal care and procedures were carried out in accordance with institutional guidelines approved by the VA Palo Alto Committee on Animal Research.

Heterochronic parabiosis was conducted as previously described ${ }^{6,13,52}$ with 3 - and 18- 
month-old mice. Briefly, incisions in the flank were made through the skin and peritoneal cavity of both mice, and adjacent peritoneal cavities were sutured together. Adjacent knee and elbow joints were then sutured together to facilitate coordinated locomotion. Skin was then stapled together using surgical autoclips (9-mm, Clay Adams), and mice were placed under heat lamps to recover from anesthesia. Each individual mouse was injected subcutaneously with Baytril antibiotic (5 $\mu \mathrm{g}$ perg) and buprenorphine $(0.05-0.1 \mu \mathrm{g} \mathrm{ml}-1)$ for pain management, and $0.9 \%(\mathrm{w} / \mathrm{v}) \mathrm{NaCl}$ for hydration. Mice were monitored and administrated drugs and saline over the next week as previously described.

EDTA-plasma was isolated by centrifugation at $1,000 \mathrm{~g}$ for $10 \mathrm{~min}$ at $4{ }^{\circ} \mathrm{C}$ before aliquoting and storing at $-80^{\circ} \mathrm{C}$. A total of 110 plasma samples were analyzed and aliquots of $150 \mu l$ of plasma were sent on dry ice to SomaLogic Inc. (Boulder, Colorado, US). Samples were sent in two different batches, 29 samples in 2016 and 81 in 2018. Data for 1,305 SOMAmer probes were obtained and no sample or probe data were excluded. RFUs of each plasma protein were log10-transformed.

The SomaScan assay has been developed and validated for human fluids but successfully used in mouse research ${ }^{9,23}$. To understand how similar mouse and human sequences are, we downloaded all homologies between mouse and human along with sequence identifiers for each species (HOM_MouseHumanSequence.rpt) from MGI (http://www.informatics.jax.org/) as plain text files. Then, the protein reference sequences for both organisms were extracted from UniProt (https://www.uniprot.org/). On these matched sequence pairs, for each protein we computed a global pairwise sequence alignment. The alignments have been calculated by using the $R$ "Biostrings" library ${ }^{53}$. The average identity was 0.85 , supporting the use of the SomaScan assay with mouse plasma.

To determine the effect of age and sex at the protein level, we used the 81 samples from 1 month to 30 months. To this end, we fitted the following linear model:

Protein level $\sim \alpha+\beta 1$ Age $+\beta 2$ Sex $+\varepsilon$

Type II sum of squares (SS) were calculated and Q-values were estimated using BenjaminiHochberg approach.

To characterize the effects of young and old blood on the aging plasma proteome, normed scaled Principal Component Analysis (PCA) was performed using the R ade $4^{54}$ package

\section{Prediction of human chronological age using the plasma proteome}

To determine whether the plasma proteome could predict chronological age, we used glmnet ${ }^{55}$ and fitted a LASSO model (alpha=1, 100 lambda tested, "lamda.min" as the shrinkage variable estimated after 10-fold CV). Input variables consisted in Z-scaled log10 RFUs and sex information. Two-thirds $(n=2,858)$ of the INTERVAL and LonGenity samples were used for training the model and the remaining 1,473 samples were used as a validation. In addition, the 171 samples from the 4 small independent cohorts were used to further assess the robustness of the predictive model.

To estimate whether a subset of the aging clock can provide similar predictive results, we used a two-step approach as we described previously ${ }^{56}$. One hundred models (100 lambda) including 0 to 373 proteins were created in step 1 and we estimated the accuracy of each of these model on the discovery and validation datasets, separately. Broken stick regression was used to determine the best compromise between number of variables and prediction accuracy.

\section{Associations between $\Delta$ age and clinical and functions in old}


We used the subjects from the LonGenity cohort to identify associations between deviations from the proteomic clock ( $\Delta$ age=predicted age-chronological age) and 334 clinical and functional variables (Supplementary Table 8). To this end, we tested the following linear model:

$$
\text { VariableOfInterest } \sim \alpha+\beta 1 \text { Age }+\beta 2 \Delta \text { Age }+\beta 3 \text { Sex }+\varepsilon
$$

For binary outcomes, logistic regression was used. This analysis was separately performed in the discovery $(n=2,858)$ and validation cohorts $(n=1,473)$. Type II sum of squares were calculated using the Anova function of the $\mathrm{R}$ car package ${ }^{43}$.

\section{Clustering of protein trajectories}

To estimate protein trajectories during aging, plasma proteins levels were z-scored and LOESS (locally estimated scatterplot smoothing) regression was fitted for each plasma factor. To group proteins with similar trajectories, pairwise differences between LOESS estimates were calculated based on the Euclidian distance and hierarchical clustering was performed using the complete method. To understand the biological functions of each cluster, we queried Reactome, KEGG and GO databases, as described above.

\section{Differential Expression - Sliding Window Analysis (DE-SWAN)}

To identify and quantify linear and non-linear changes of the plasma proteome during aging, we developed a Differential Expression - Sliding Window ANalysis (DE-SWAN) approach. Considering, a vector $I$ of $k$ unique ages, we iteratively used $I_{k}$ as the center of a 20-year window and compared protein levels of subjects in parcels above and below $l_{\mathrm{k}}$ i.e. $\left[l_{k}-10 \mathrm{y} ; l_{k}[\mathrm{vs}\right.$ ]$\left.l_{k} ; l_{k}+10 \mathrm{y}\right]$. To test for differential expression, we used the following linear model:

$$
\text { Protein level } \sim \alpha+\beta 1 \text { Age }_{\text {Low } / \mathrm{High}}+\beta 2 \mathrm{Sex}+\varepsilon
$$

Age being binarized according to the parcels. For each $\mathrm{l}_{\mathrm{k}}$, $\mathrm{q}$-values were estimated using Benjamini-Hochberg correction. Type II sum of squares were calculated using the Anova function of the R car package ${ }^{43}$.

To assess the robustness and relevance of DE-SWAN results, we tested multiple parcel widths (5, 10, 15 and 20 years). In addition, we used multiple q-values thresholds and compared these results with those obtained by chance. To this end, we randomly permutated the phenotypes of the subjects and applied DE-SWAN to this new dataset. To keep the data structure, age and sex were permuted together. In addition, we analyzed the INTERVAL and LonGenity cohorts separately (Supplementary Fig. 6). Finally, we tested the same linear model when adjusting for Subcohort. This led to a loss of statistical power when the age range of the INTERVAL and LonGenity cohorts were overlapping but the three waves of aging proteins remained and the ranks of the top proteins were nearly identical (Supplementary Fig. 8). We used the model adjusted for subcohort when trying to understand the waves of aging proteins. The significance levels of the intersections between aging plasma protein signatures identified by linear modeling and DESWAN at different ages were determined using the R SuperExactTest package ${ }^{57}$.

Relationships between the aging waves and the genome and the proteome of disease and
traits. traits.

To quantify the overlap between proteins changing with age at different stages of life and the genome and the proteome of diseases and traits, we ranked DE-SWAN results based on pvalues and created a k-ranked list of aging proteins, $L_{k}$. To reflect the degree to which the 
genome/proteome are linked with the waves of aging plasma proteins, we walked down $L_{k}$ and counted the number of proteins associated with the genome or specific proteome. When different versions of the SomaScan platform were used, we walked down $L_{k}$ until reaching the top 100 proteins measured in both studies.

To identify specific genetic variants associated with the aging plasma proteome, we mined the summary statistics generated by Sun et al. ${ }^{25}$, who found 1,927 associations with 1,104 plasma proteins. Qgraph ${ }^{58}$ R package was used to create a network between the genome and the 2,925 proteins analyzed in this study.

To determine whether the aging proteome is associated with the proteome of clinical and functional variables, we used the subjects from the LonGenity cohort and tested the following linear model for the top variables identified in Supplementary Table 8:

Protein level $\sim \alpha+\beta 1$ Age $+\beta 2$ Sex $+\beta 3$ VariableOfInterest $+\varepsilon$

Type II sum of squares were calculated using the Anova function of the $R$ car package ${ }^{43}$.

To determine whether the aging proteome is associated with disease proteomes, we integrated data and results from previous proteomic studies using the SomaScan platform. We re-analyzed one Alzheimer's Disease - AD dataset publicly available by AddNeuroMed ${ }^{26}$ and used summary statistics from published studies focused cardiovascular disease - CVD ${ }^{28}$, Down Syndrom - $\mathrm{DS}^{27}$ and body mass index $-\mathrm{BMI}^{29}$.

AddNeuroMed is a European multi-center study in which the AD proteome was quantified in plasma samples from 681 controls, Mild Cognitive Impairment (MCI), and AD subjects using a previous version of the SomaScan assay. Files used were downloaded from the Synapse portal in March 2016 (syn5367752) and included measurements of 1016 plasma proteins from 931 samples. We limited our analysis to the 645 samples available at visit 1 (191 control, $165 \mathrm{MCl}$ and 289 AD). Raw data were log10-transformed. Four samples (2 control and 2 AD) were considered as outliers based on principal component analysis and filtered out.

To identify plasma proteins associated with $A D$, we used linear models with diagnosis, age, sex and center as covariates:

$$
\text { Protein level } \sim \alpha+\beta 1 \text { Diagnosis }+\beta 2 \text { Age }+\beta 3 \text { Sex }+\beta 4 \text { Center }+\varepsilon
$$

Type II sum of squares were calculated using the Anova function of the R car package ${ }^{43}$.

To determine whether aging plasma proteins are involved in other disease signatures, we identified three studies using the SomaScan platform in large human cohorts providing detailed summary statistics. Carayol et al. mined the plasma proteome to obtain new insights into the molecular mechanisms of obesity. Out of 1,129 proteins measured, they identified 192 plasma proteins significantly associated with BMI ( $p<0.05$ after Bonferroni correction). Summary statistics we used were obtained from their "Supplementary Data 1"29. Ganz et al. derived and validated a 9 protein risk-score to predict risk of cardiovascular outcomes ${ }^{28}$. In addition to these 9 proteins, 191 other proteins were significantly associated with cardiovascular risk $(p<0.05$ after Bonferroni correction). Summary statistics for these 200 proteins are available in their eTable 4 and the 1,130 proteins measured of this study are listed in their eTable 1. Finally, Sullivan et al. used an extended version of the SomaScan platform to study DS and identified a large number of dysregulated proteins ${ }^{27}$. We used the results for the Discovery cohort (sheet $A$ of the "Supplementary File 1") in which, 258 proteins out of 3,586 proteins are reported to be associated with DS ( $p<0.05$ after Bonferroni correction).

Since detailed protein information was not available for all studies, we used either gene symbols or unitprotID to merge disease proteomes characterized in published studies with the aging proteome identified in this study. When multiple $p$-values were reported for the same gene symbol (or a combination of gene symbols), only the most significant $p$-value was retained.

\section{Code availability}


An $R$ package for DE-SWAN is available in github:

https://github.com/lehallib/DEswan

\section{Data availability.}

We created a searchable web interface to mine the human INTERVAL and LonGenity datasets (https://twc-stanford.shinyapps.io/aging plasma proteome/)

The Human independent cohorts and mouse protein data are available in Supplementary Tables 16 and 17. The Interval data is available through the European Genome-Phenome Archive (https://ega-archive.org/studies/EGAS00001002555). 
bioRxiv preprint doi: https://doi.org/10.1101/751115; this version posted September 1, 2019. The copyright holder for this preprint (which was not certified by peer review) is the author/funder. All rights reserved. No reuse allowed without permission.

\section{Figures and Legends}



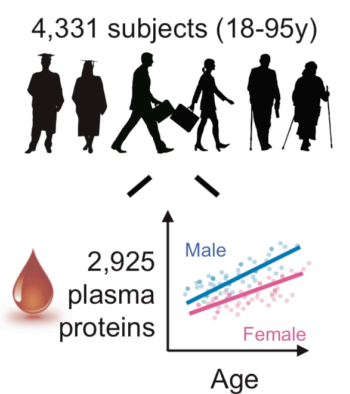

d

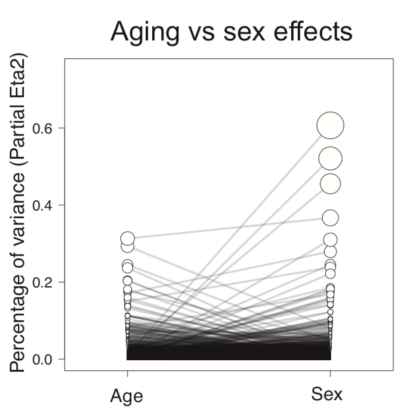

b

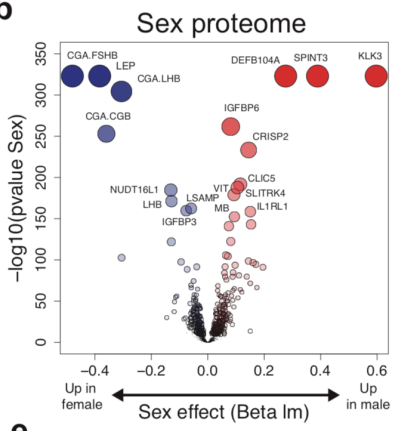

e

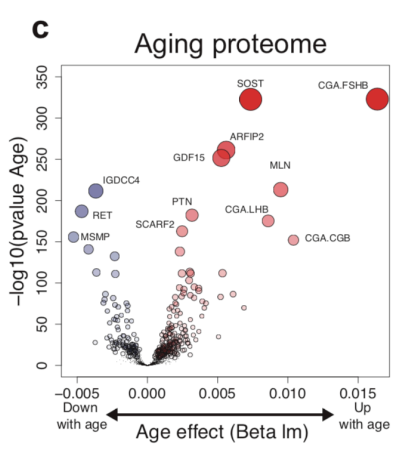

Figure 1

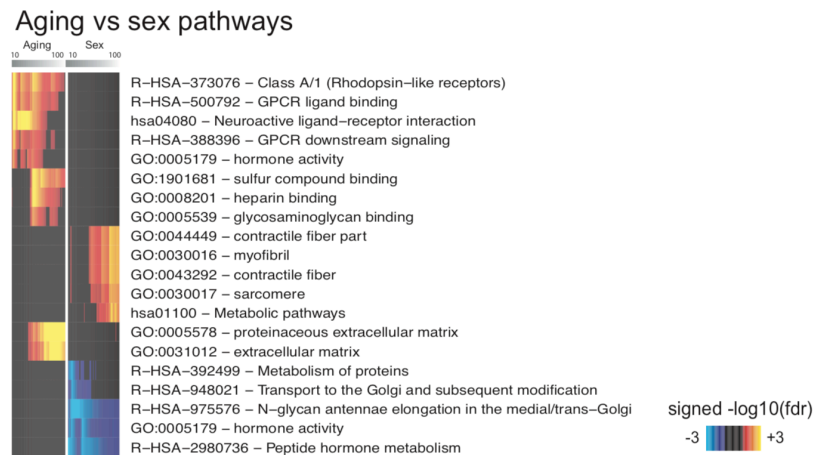

f

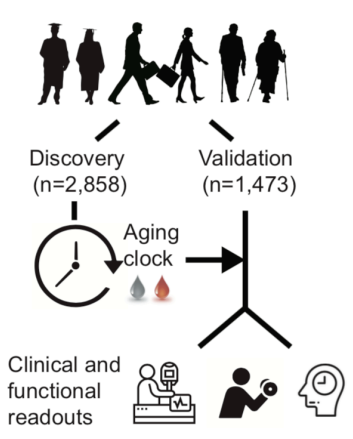

i

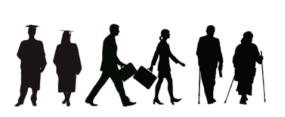

Human aging proteome

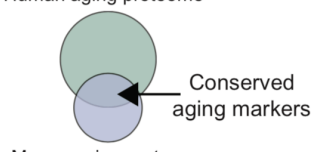

Mouse aging proteome

तु हैं g

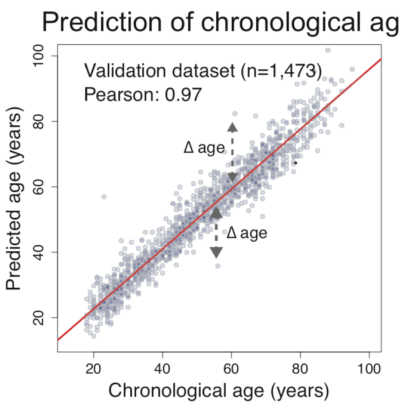

\section{j}

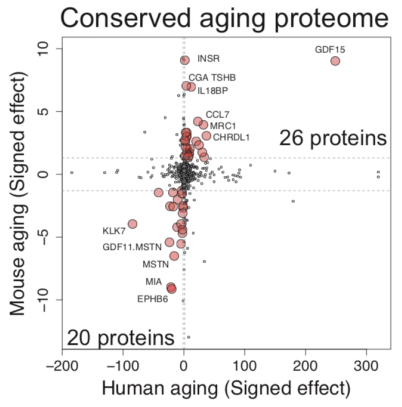

h

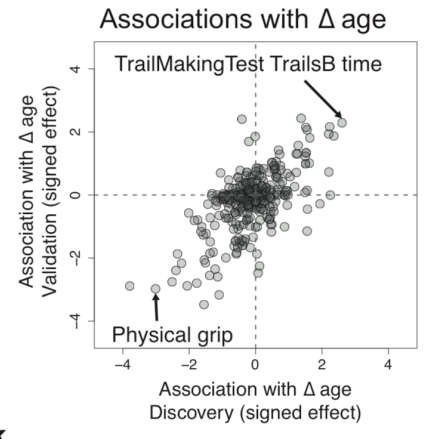

k

Alteration of the conserved aging signature by parabiosis

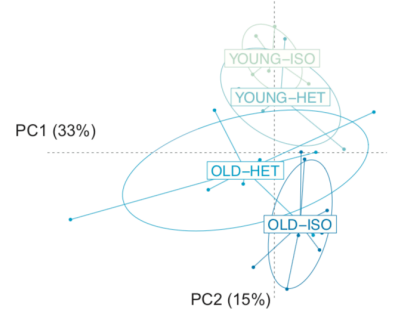

I

Non-uniform changes of the conserved aging signature

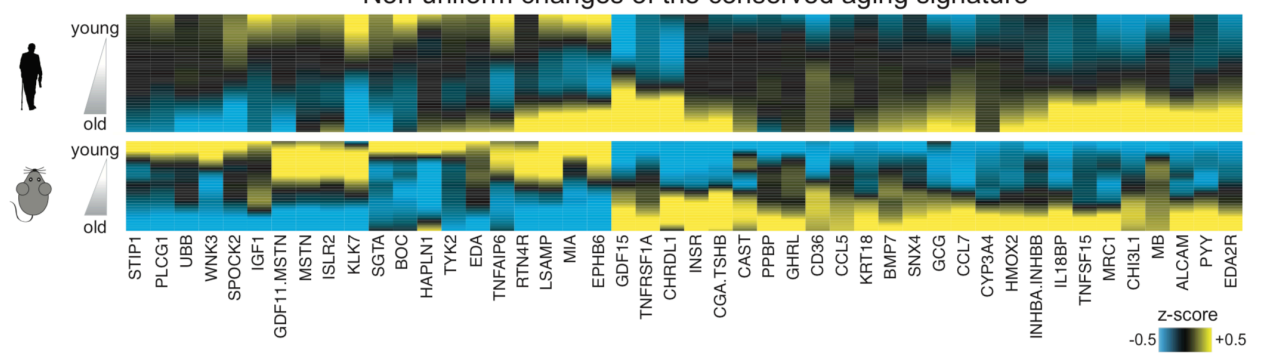


Figure 1: Linear modeling links the plasma proteome to functional aging and identifies a conserved aging signature.

(a) Schematic representation of the linear analysis of the plasma proteome. Four thousand three hundred thirty-one human EDTA plasma samples were measured using the SomaScan platform and analyzed using linear models, adjusted for age, sex and subcohort.

Volcano plots representing changes of the plasma proteome with sex (b) and age (c). Dot sizes are proportional to the product of $-\log 10$ (p-value) and sex or age effect (beta of the linear model).

(d) Relative percentage of variance explained by age and sex. Partial Eta2 is calculated for age and sex. Values for each plasma protein are connected by edges.

(e) Pathways associated with sex and age identified by Sliding Enrichment Pathway Analysis (SEPA). Enrichment for pathways in the top 10 to top 100 proteins ranked by the product of $\log 10$ ( $p$-value) and beta $\mathrm{Im}$ is tested, in increments of 1 protein. Proteins upregulated and downregulated are analyzed separately. The top 10 pathways per condition are represented.

(f) Modeling chronological age using the plasma proteome analysis. A LASSO model with 10-fold cross validation was trained on $2 / 3$ of the subjects $(n=2,858$ out of 4,331 ). The model was tested on the remaining 1,473 samples. Then, association between delta age (difference between predicted age and chronological age) with functional readouts was investigated.

(g) Prediction of age in the validation cohort $(n=1,473)$ using 373 plasma markers. Pearson correlation coefficient between observed and predicted age is indicated.

(h) Association between delta age (difference between observed age and chronological age) and functional readouts in old. Linear modeling between 334 functional readouts and delta age adjusted for age at clinical visit, sex and cohort was tested. Top associations in both discovery and validation datasets are represented.

(i) Schematic representation of the comparison between the human and mouse aging proteomes.

(j) Conserved markers of aging. Effect of aging on the human plasma proteome is compared to the effect of aging on the mouse plasma proteome. Both human and mouse aging effects are signed by the beta age of their corresponding linear analysis. Forty-six plasma proteins are changing in the same direction in mouse and humans (red dots) and define a conserved aging signature.

(k) Alteration of the conserved aging signature by parabiosis. Normed principal component analysis was used to characterize changes of the conserved aging signature when mice are exposed to young or old blood.

(I) Age-related changes of the conserved aging signature. Plasma protein levels were z-scored and aging trajectories were estimated by locally estimated scatterplot smoothing (LOESS). 
Figure 2

a

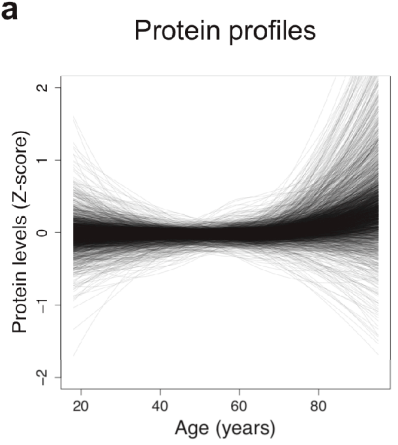

b

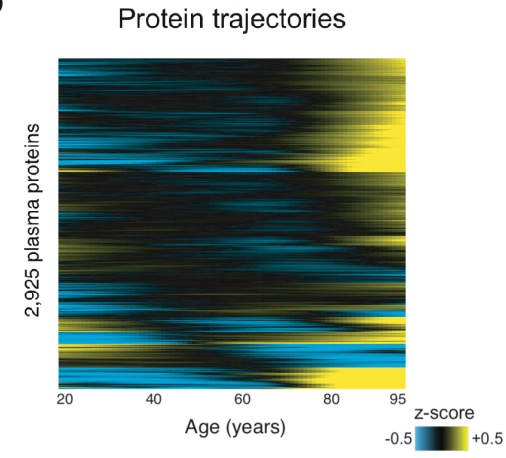

Cluster trajectories

d
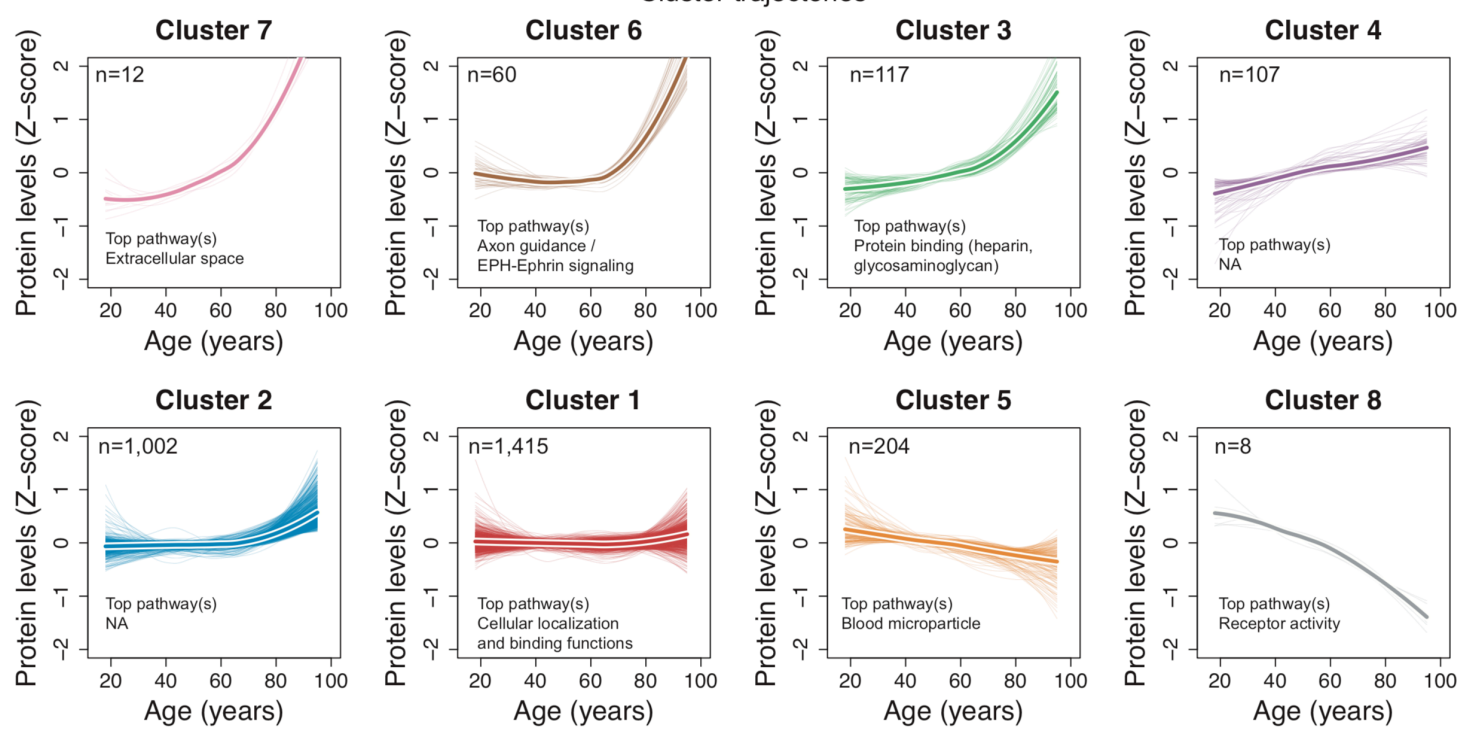

Figure 2: Clustering of protein trajectories identifies linear and non-linear changes during aging.

(a) Protein trajectories during aging. Plasma protein levels were z-scored and trajectories of the 2,925 plasma proteins were estimated by LOESS.

(b) Trajectories are represented in two dimensions by a heatmap and unsupervised hierarchical clustering was used to group plasma proteins with similar trajectories.

(c) Hierarchical clustering dendrogram. The 8 clusters identified are represented by orange boxes.

(d) Protein trajectories of the 8 identified clusters. Clusters are grouped by the similarity of global trajectories, the thicker lines representing the average trajectory for each cluster. The number of proteins and the most significant enriched pathways are represented for each cluster. Pathway enrichment was tested using GO, Reactome and KEGG databases. The top pathways are indicated and the top 20 pathways for each cluster are listed in supplementary table 12. 
Figure 3

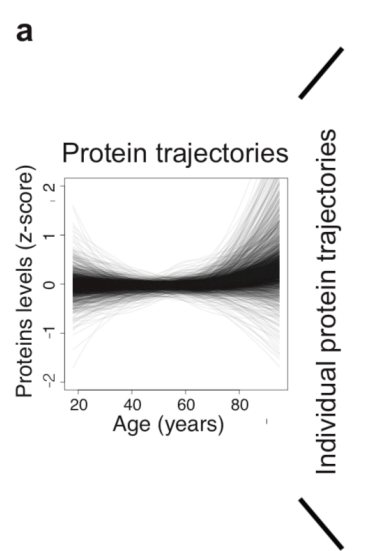

Differential Expression (DE) - Sliding Window ANalysis (SWAN)

b

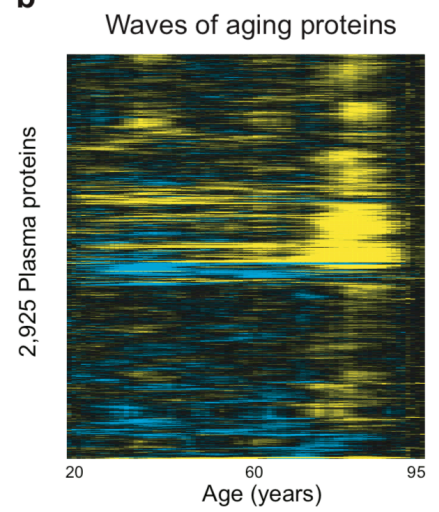

e

Overlap between waves of aging proteins

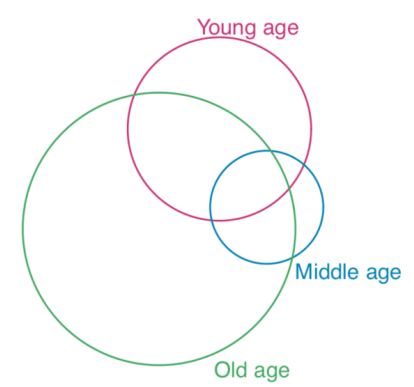

f Overlap between aging waves and linear modeling 600 .

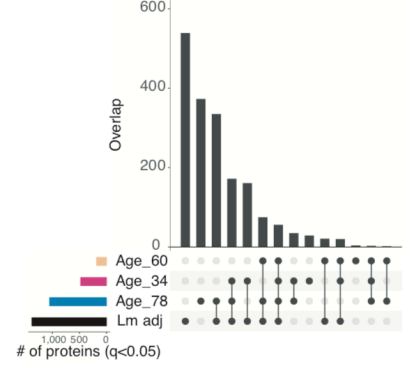

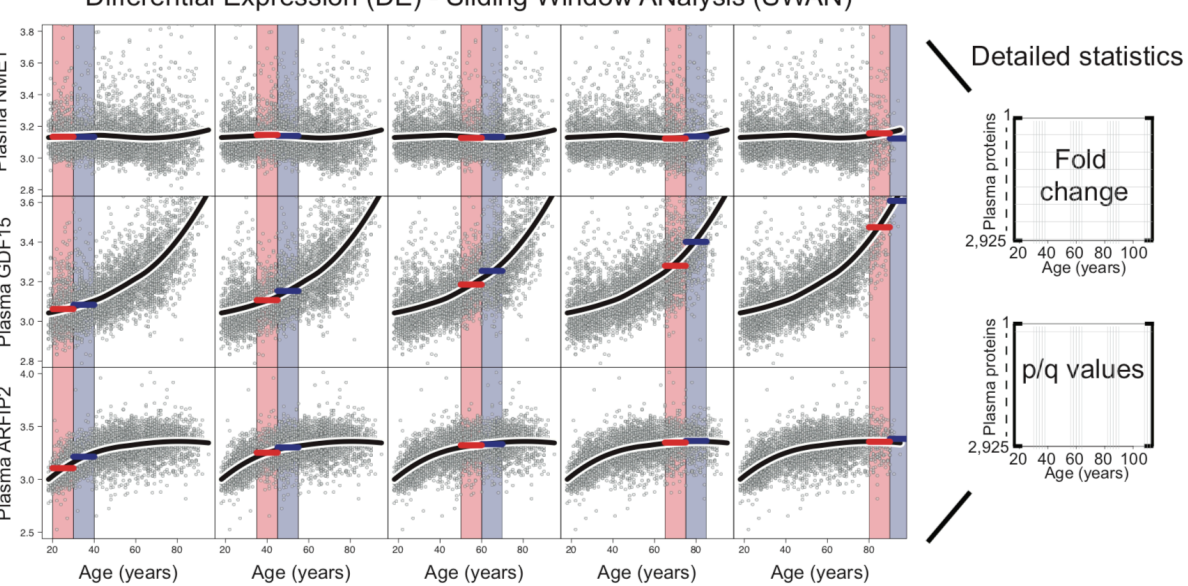

c

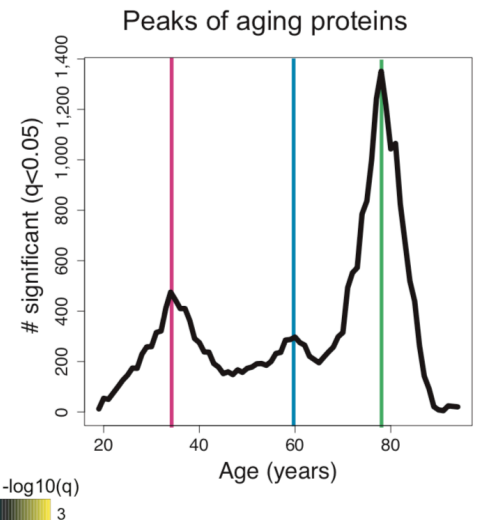

d

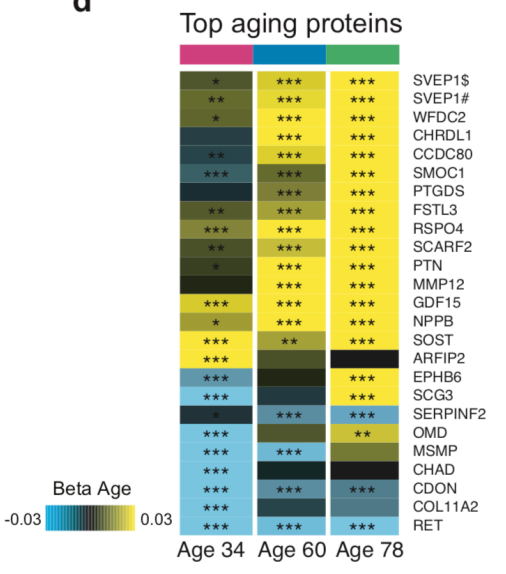

g Aging pathways Lm Age 34 Age $60 \quad$ Age 78

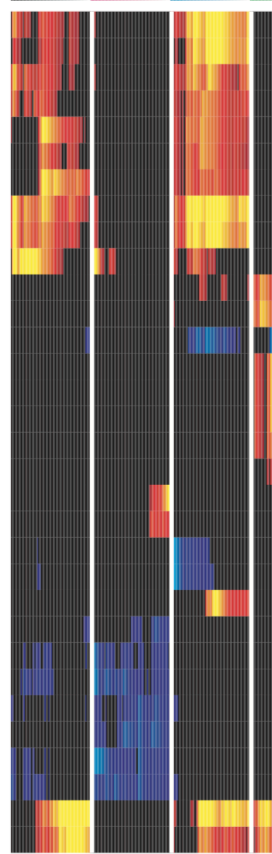

signed $-\log 10(\mathrm{fdr})$

-3 ind +3

R-HSA-375276 - Peptide ligand-binding receptors R-HSA-418594 - G alpha (i) signalling events R-HSA-388396 - GPCR downstream signaling GO:0005179 - hormone activity GO:0008201 - heparin binding GO:0005539 - glycosaminoglycan binding GO:1901681 - sulfur compound binding

R-HSA-373076 - Class A/1 (Rhodopsin-like receptors) R-HSA-500792 - GPCR ligand binding

hsa04080 - Neuroactive ligand-receptor interaction GO:0044420 - extracellular matrix component GO:0005604 - basement membrane

GO:0072562 - blood microparticle

GO:0071772 - response to BMP

GO:0030509 - BMP signaling pathway GO:0071773 - cellular response to BMP stimulus GO:0030514 - negative regulation of BMP signaling pathway

GO:0070848 - response to growth factor GO:0003723 - RNA binding

GO:0017076 - purine nucleotide binding

GO:0019199 - transmembrane receptor protein kinase activity

GO:0004714 - transmembrane receptor protein tyrosine kinase activity

GO:0009617 - response to bacterium

R-HSA-3000178 - ECM proteoglycans

GO:0005578 - proteinaceous extracellular matrix GO:0031012 - extracellular matrix

R-HSA-1474244 - Extracellular matrix organization R-HSA-3000171 - Non-integrin membrane-ECM interactions

R-HSA-2022090 - Assembly of collagen fibrils \& other multimeric structures R-HSA-1474228 - Degradation of the extracellular matrix GO:0005578 - proteinaceous extracellular matrix

GO:0031012 - extracellular matrix 
Figure 3: Sliding window analysis distinguishes waves of aging plasma factors. (a) Principles of Differential Expression Sliding Window Analysis (DE-SWAN) compares levels of plasma proteins between groups of individuals in parcels of 10 years, e.g. 30-40 compared with 40-50. By sliding the window, DE-SWAN scans the whole aging plasma proteome and identifies linear and non-linear changes with age. Examples of the DE-SWAN approach for 3 plasma proteins and 5 age windows are represented. Red and blue rectangles show the two parcels that are compared and the red and blue lines symbolize the mean within each parcel. DE-SWAN provides statistics for each age window and each plasma protein, allowing a detailed analysis of plasma proteomic changes during aging.

(b) Waves of aging plasma proteome characterized by DE-SWAN. Within each window, - $\log 10(p-$ values) and -log10(q-values) were estimated by linear modeling adjusted for age and sex. Local changes attributable to age were signed based on corresponding beta age.

(c) Number of plasma proteins differentially expressed during aging. Three local peaks at the ages of 34,60 and 78 were identified by DE-SWAN.

(d) Top 10 plasma proteins identified by linear modeling and DE-SWAN at age 34, 60 and 78. Blue and yellow colors represent local increase and decrease, respectively. \# and $\$$ indicate different SOMAmers targeting the same protein. ${ }^{*} \mathrm{q}<0.05$, ${ }^{* *} \mathrm{q}<0.01,{ }^{* * *} \mathrm{q}<0.001$

(e) Intersections between aging plasma proteins identified by linear modeling and DE-SWAN at ages 34,60 and $78(q<0.05)$ visualized by Venn diagram.

(f) Intersections between the waves and linear modeling $(q<0.05)$ visualized by Upset plot.

(g) Visualization of pathways significantly enriched for aging proteins identified by linear modeling and DE-SWAN at age 34, 60 and 78. Enrichment in the top 10 to top 100 proteins were tested in increments of 1 protein. Proteins upregulated and downregulated were analyzed separately. GO, Reactome and KEGG databases are shown. The top 10 pathways per condition are represented. 
a

Relevance of the aging waves

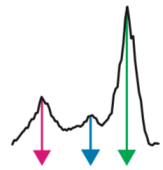

Proteins ranked by

pvalue at 34,60 and $78 y$

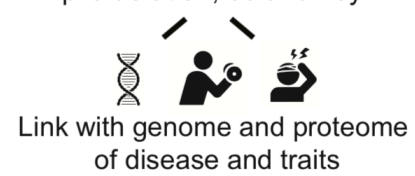

d

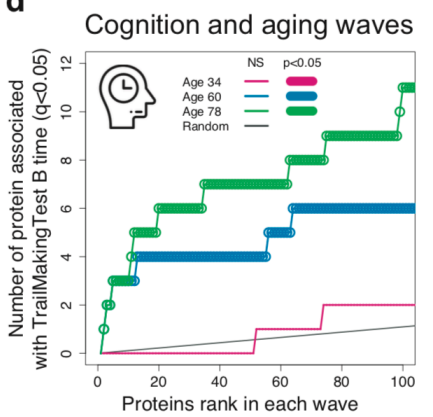

g

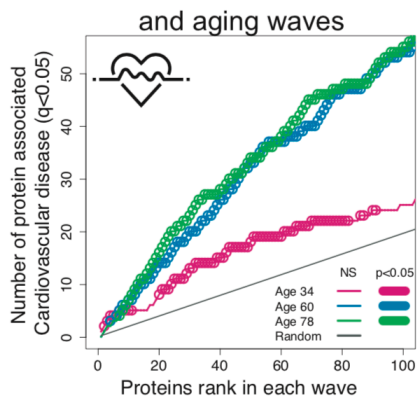

b

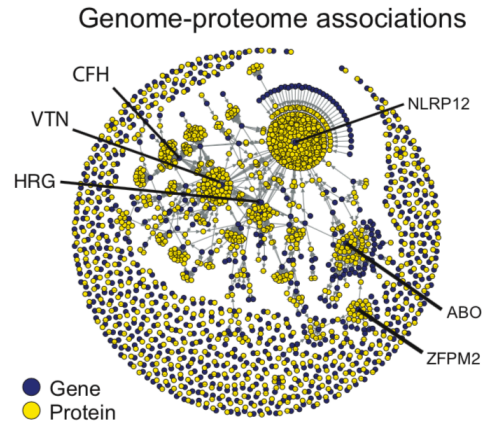

e

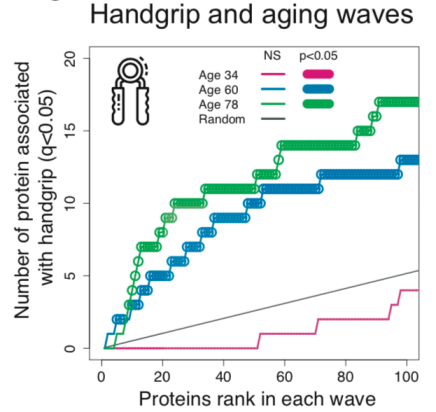

h Alzheimer's disease

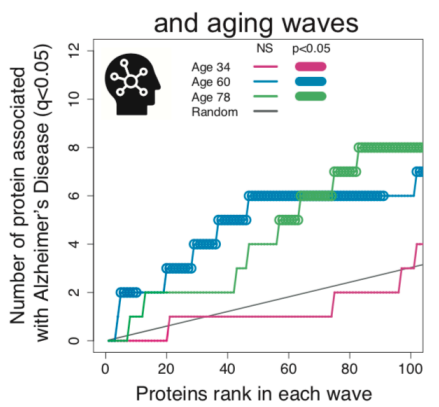

C

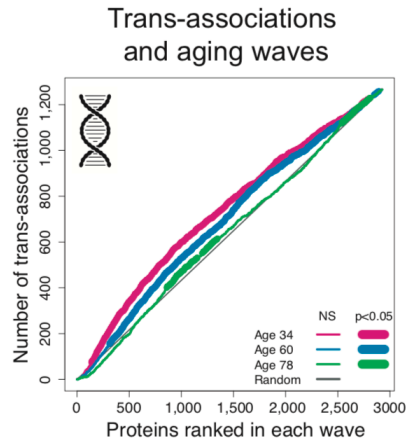

$\mathbf{f}$

Body Mass Index

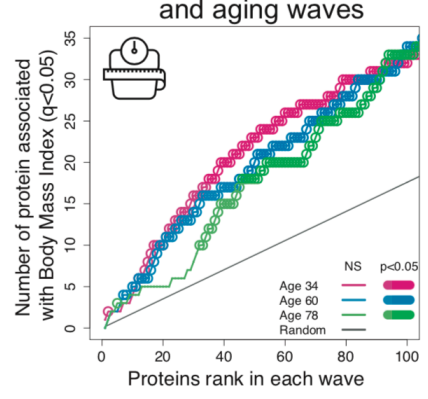

i

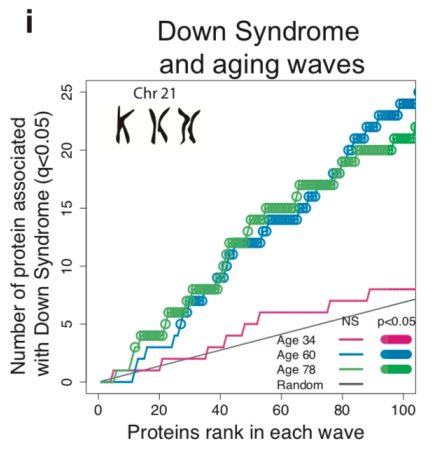

Figure 4: Waves of aging proteins are differentially linked to the genome and proteome of disease and traits

(a) Relevance of the aging waves. Schematic representation of analysis. The proteins changing at 34,60 and $78 y$ were ranked by $p$-value and were associated with the genome and the proteome of disease and traits.

(b) Association between the genome and the proteome. Network created using the pQTL associations identified by Sun et al. (2018).

(c) Enrichment for trans-association in the waves of aging proteins identified by DE-SWAN. Aging proteins at age 34, 60 and $78 y$ were ranked based on p-value and the cumulative number of transassociations was enumerated. Permutation based tests $(1 e+5)$ were used to assess significance. Enrichment for proteins involved in cognitive and physical performance in the waves of aging proteins identified (d-e). Enrichment for disease-associated proteins in the waves of aging proteins $(\mathrm{f}-\mathrm{i})$. 
S1) Sample demographics

a

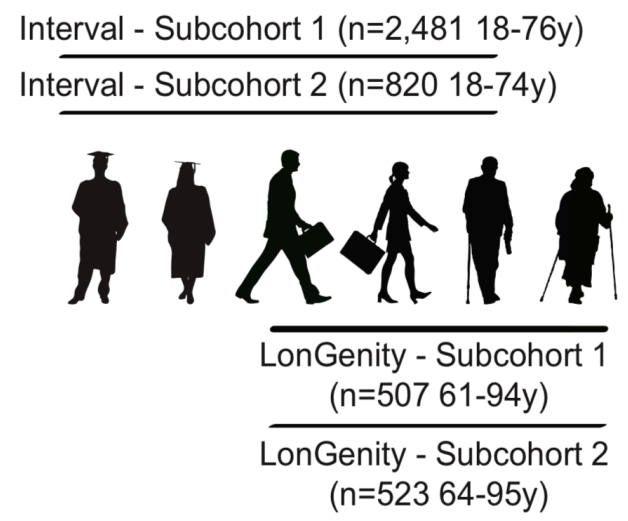

C

Female $(n=2,189)$

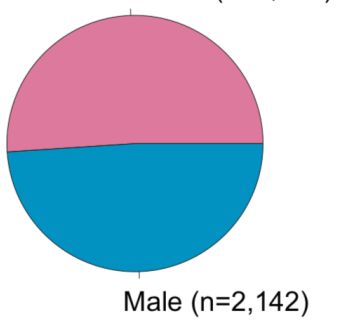

b

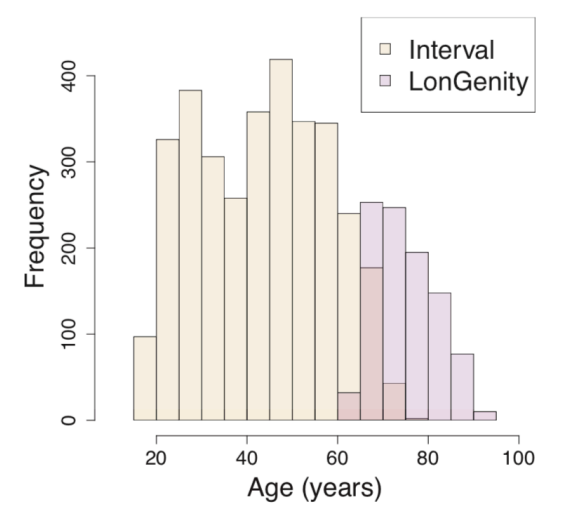

d GEHA(n=26, 89-104y) PRIN09 (n=24, 56-107y) PRIN06 (n=51, 50-102y) VA Seattle $(n=70,21-88 y)$

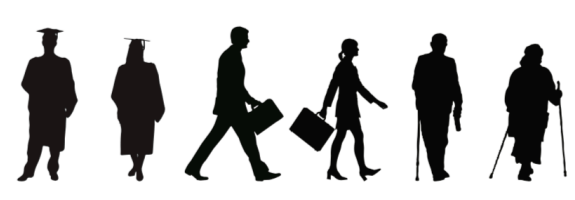

Supplementary Figure 1: Sample demographics.

Age $(a, b)$, cohort $(a, b)$ and sex distributions (c) of the 4,331 subjects from the Interval and LonGenity cohorts. (d) Age and cohort distributions of the 171 subjects from the 4 independent cohorts. 
S2) Comparing sex and aging effects in independent cohorts and studies

a

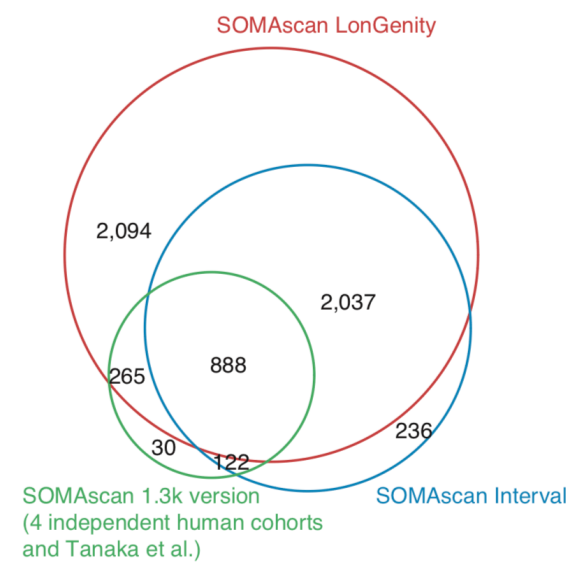

C

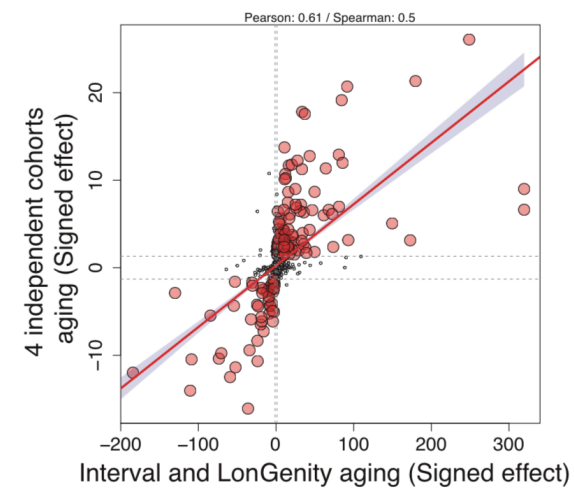

b

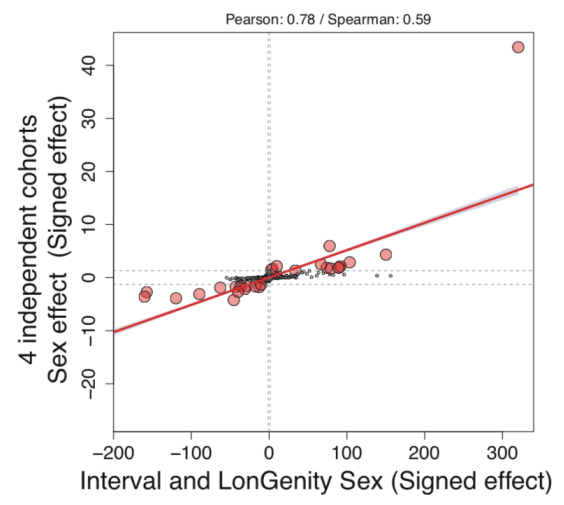

d

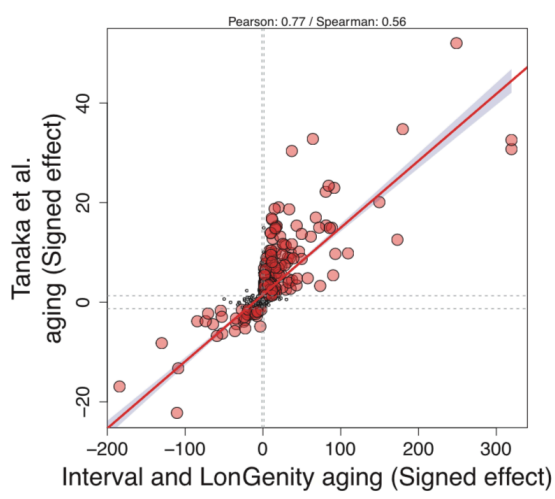

Supplementary Figure 2: Comparing aging effect in independent cohorts and studies.

(a) Age and sex effects in the INTERVAL and LonGenity studies were compared to age and sex effects in 4 independent cohorts analyzed together and to age effect from Tanaka et al. (2018) The aging plasma proteome was measured with the SomaScan assay in these cohorts and 888 proteins were measured in all studies

(b) Scatter plot representing the signed $-\log 10$ (q value) of the sex effect in the INTERVAL/LonGenity cohorts ( $x$ axis) vs the 4 independent cohorts ( $y$-axis).

Similar analysis for the age effect in the 4 independent cohorts (c) and in Tanaka et al (2018) study (d) 
S3) Deeper investigation of the aging plasma proteomic clock

a

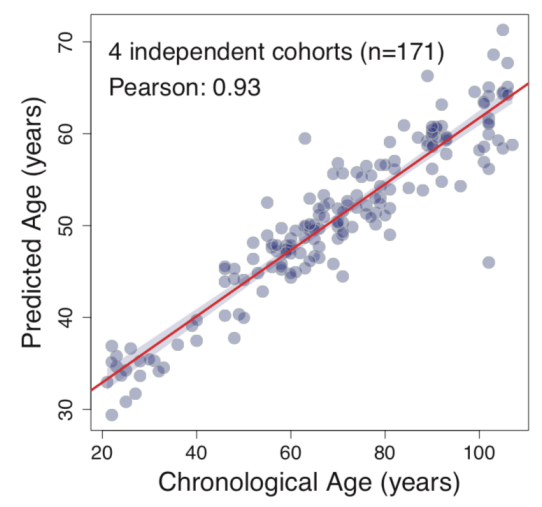

C

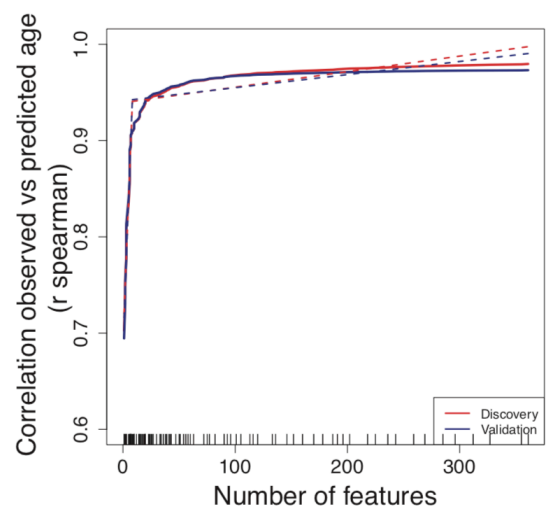

b

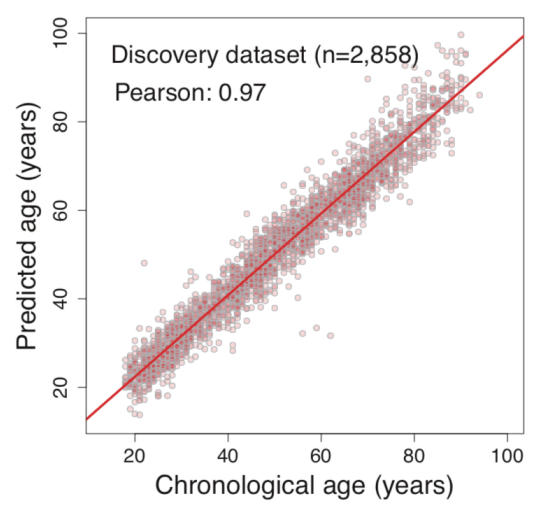

d

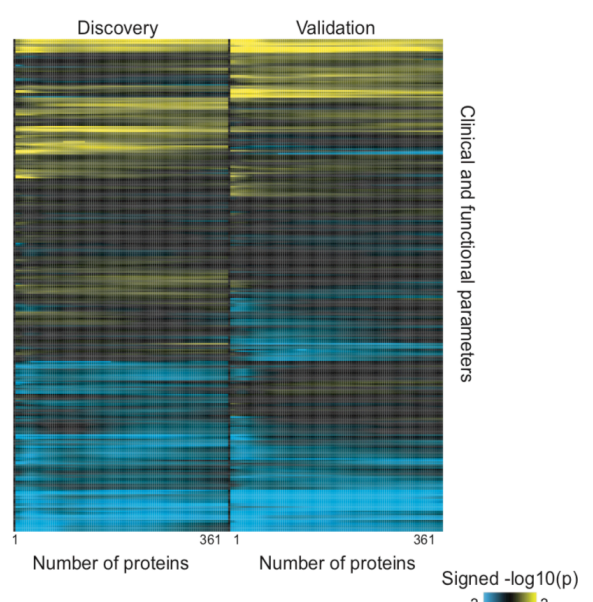

Signed $-\log 10(p)$

Supplementary Figure 3: Deeper investigation of the aging proteomic clock. (a) Prediction of age in the 4 independent cohorts $(n=171)$ using the proteomic clock. Only 141 proteins out of the 373 constituting the clock were measured in these samples. (b) Prediction of age in the discovery cohort $(n=2,858)$ using 373 plasma markers.

(c) Feature reduction of the aging model. A two-step approach was used to estimate whether a subset of the aging signature can provide similar results to the 373 aging proteins. The proteins were ranked according to the absolute value of their coefficients in the LASSO model. Then, a ridge regression model was built using the discovery dataset with the top 2 variables selected in step 1 . This process was then repeated iteratively using the top $3,4,[\ldots]$ up to top 373 variables selected in the first step. Dashed lines represent a broken stick model and indicate the best compromise between number of variables and prediction accuracy.

(d) Heatmap representing the associations between delta age and 334 clinical and functional variables. As in (c) the analysis was performed for the top 2 to top 373 variables predicting 

not certified by peer review) is the author/funder. All rights reserved. No reuse allowed without permission.

chronological age. The non-uniformity in the heatmaps suggests that specific subsets of proteins may best predict certain clinical and functional parameters. 
S4) Proteins and proteome undulations in independent human cohorts and in mouse

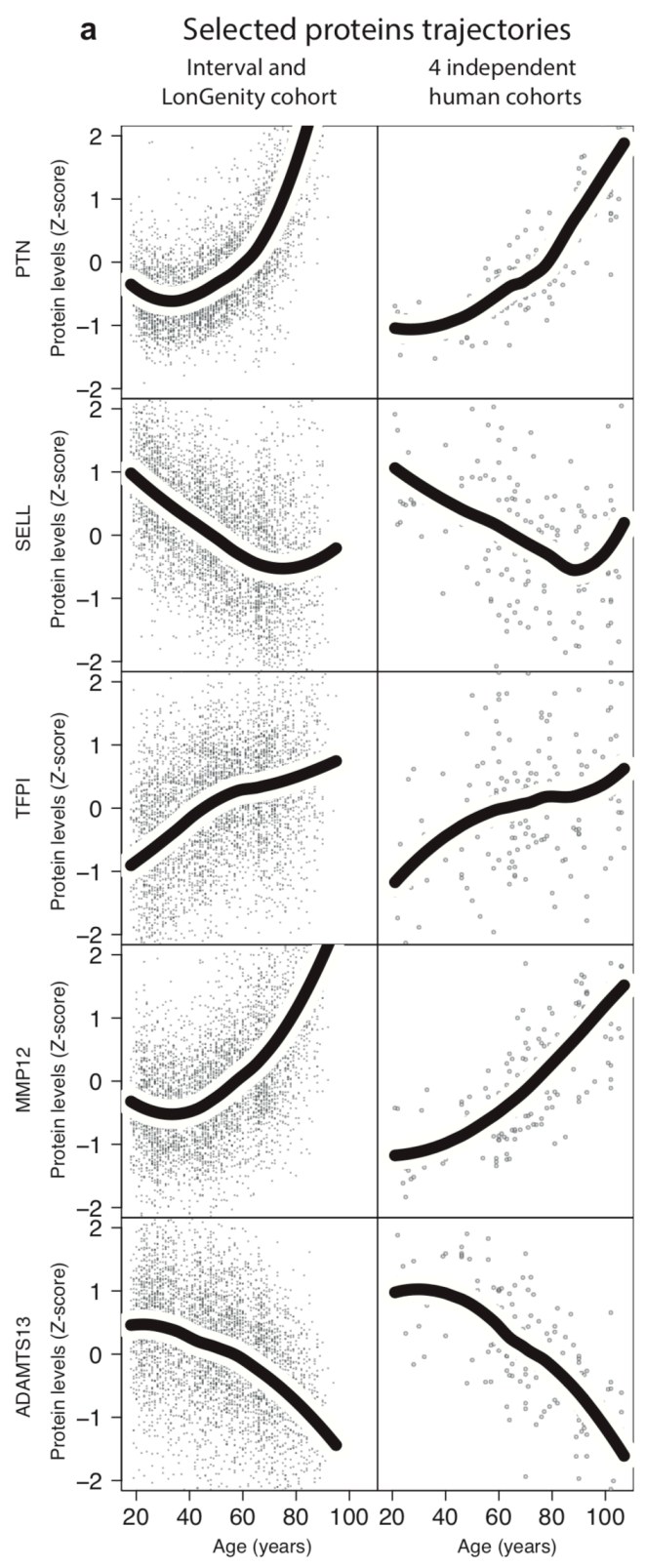

b Proteome trajectories in independent cohorts

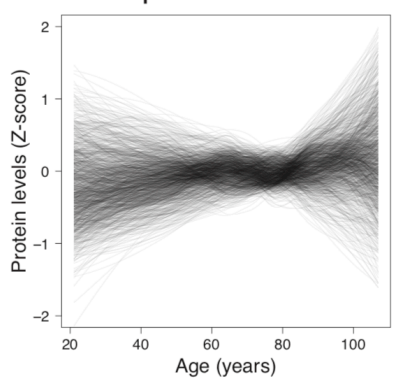

Proteome trajectories in mouse

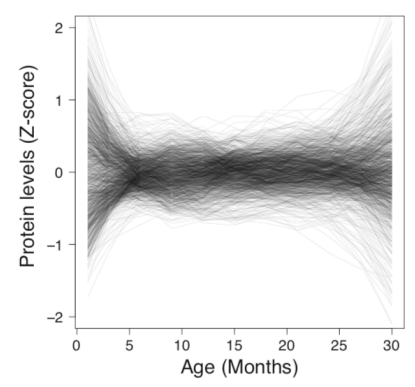

Supplementary Figure 4: Proteins and proteome undulations in independent human 
cohorts and in mouse. (a) Trajectories of 5 selected proteins based on the Interval and LonGenity cohorts ( $n=4,331$, left) and 4 independent human cohorts $(n=171$, right). Trajectories were estimated using LOESS regression.

(b) Undulation of the 1,305 plasma proteins in 4 independent cohorts ( $n=171$, left) and in mouse $(n=81$, right). Plasma proteins levels were $z$-scored and LOESS regression was fitted for each plasma factor. 
S5) Cluster trajectories in independent cohorts

Cluster 7

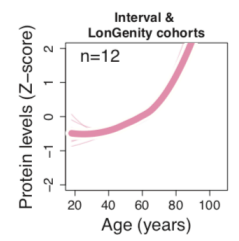

Cluster 6

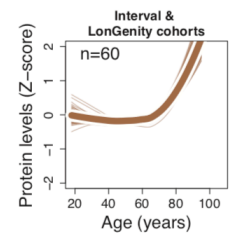

Cluster 3

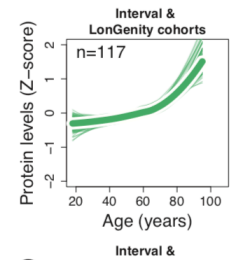

Cluster 4

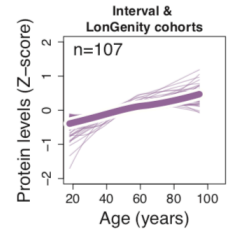

Cluster 2

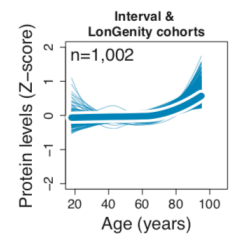

Cluster 1

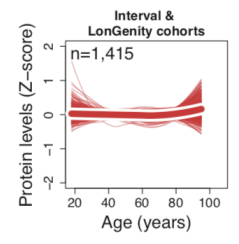

Cluster 5

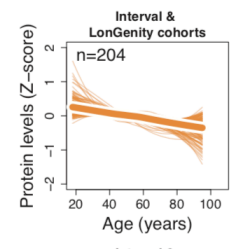

Cluster 8

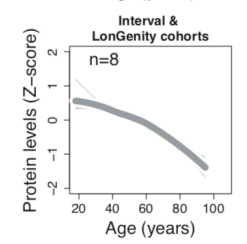

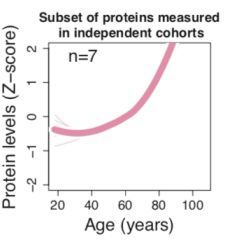
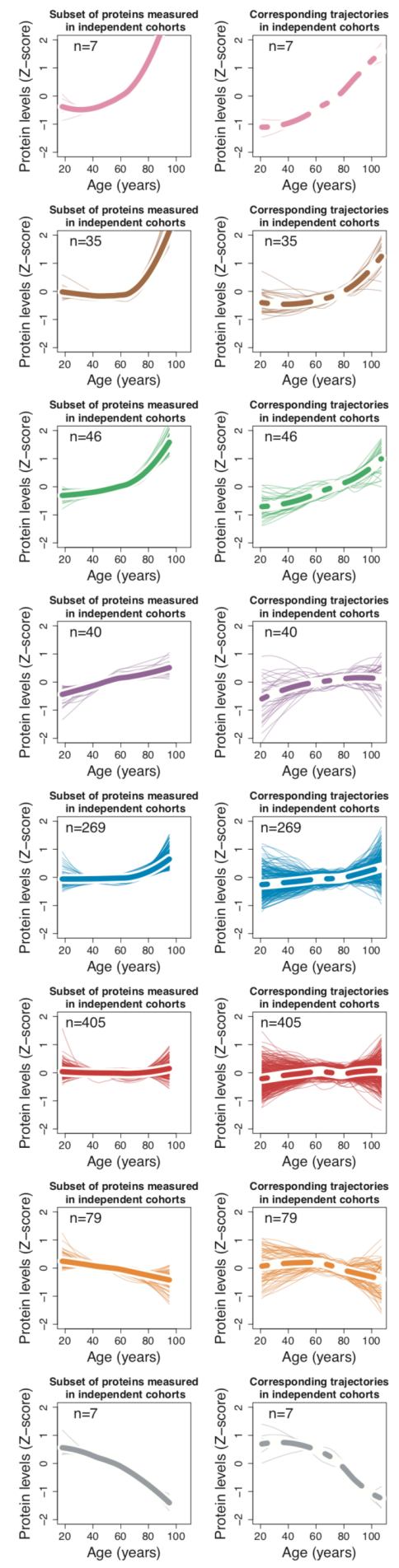

Supplementary Figure 5: Cluster trajectories in independent cohorts. Protein trajectories for the 8 clusters identified in the Interval and LonGenity cohorts (left column). Thicker lines represent the average trajectory for each cluster. Cluster trajectories for the subset of proteins 
measured in the 4 independent cohorts (middle column). Corresponding cluster trajectories in 4 independent cohorts (right column). 
S6) Top pathways in clusters

\begin{tabular}{|c|c|c|c|c|}
\hline Cluster 1 & nuclear part (319) & cytosol (437) & nucleoplasm (250) & nuclear lumen (286) \\
\hline Cluster 2 & $\begin{array}{l}\text { Human cytomegalovirus } \\
\text { infection (31) }\end{array}$ & Chronic myeloid leukemia (16) & $\begin{array}{l}\text { Cytokine-cytokine receptor } \\
\text { interaction (79) }\end{array}$ & FoxO signaling pathway (23) \\
\hline Cluster 3 & extracellular region (80) & $\begin{array}{l}\text { extracellular region part } \\
\qquad(69)\end{array}$ & extracellular space (44) & $\begin{array}{l}\text { proteinaceous extracellular } \\
\text { matrix }(16)\end{array}$ \\
\hline Cluster 4 & $\begin{array}{l}\text { Chondroitin sulfate } \\
\text { biosynthesis (4) }\end{array}$ & $\begin{array}{l}\text { Heparan sulfate/heparin } \\
\text { (HS-GAG) metabolism (5) }\end{array}$ & $\begin{array}{l}\text { Chondroitin sulfate/dermatan } \\
\text { sulfate metabolism (5) }\end{array}$ & $\begin{array}{l}\text { Regulation of IFNG signaling } \\
\text { (3) }\end{array}$ \\
\hline Cluster 5 & extracellular space (62) & blood microparticle (10) & fibrillar collagen trimer (3) & banded collagen fibril (3) \\
\hline Cluster 6 & $\begin{array}{l}\text { EPH-ephrin mediated repulsion } \\
\text { of cells (7) }\end{array}$ & EPH-Ephrin signaling (7) & $\begin{array}{l}\text { ephrin receptor signaling } \\
\text { pathway (8) }\end{array}$ & extracellular region (45) \\
\hline Cluster 7 & extracellular space (8) & fibronectin binding (2) & extracellular region (10) & extracellular region part (9) \\
\hline Cluster 8 & $\begin{array}{l}\text { transmembrane receptor } \\
\text { protein tyrosine kinase } \\
\text { activity (3) }\end{array}$ & $\begin{array}{l}\text { signal transducer, downstream } \\
\text { of receptor, with protein } \\
\text { tyrosine kinase activity (2) }\end{array}$ & integrin binding (3) & $\begin{array}{l}\text { transmembrane receptor } \\
\text { protein kinase activity (3) }\end{array}$ \\
\hline & 1 & & & 4 \\
\hline & $\begin{array}{l}\square \text { fdr }>0.1 \\
\square \text { fdr }<0.1 \\
\square \text { fdr }<0.05 \\
\square \text { fdr }<0.01\end{array}$ & \multicolumn{2}{|c|}{ Top Pathways (Rank) } & \\
\hline
\end{tabular}

Supplementary Figure 6: Pathway enrichment was tested using GO, Reactome and KEGG databases. The top 4 pathways for each cluster are shown. Pathway IDs and number of plasma proteins associated are represented in the table. 
S7) DE-SWAN age effect for multiple qvalues cutoffs, window sizes and after phenotype permutation

a

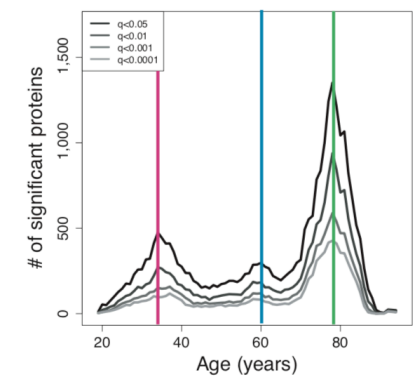

C

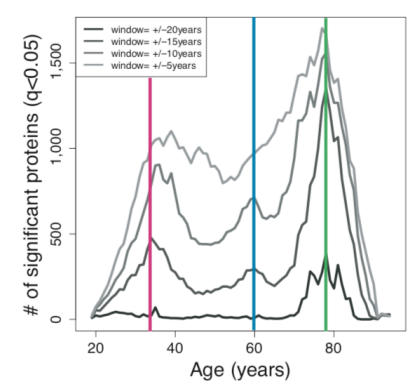

b

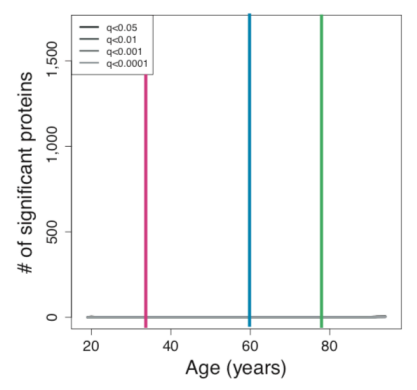

Supplementary Figure 7: DE-SWAN age effect for multiple q-values cutoffs, windows size and after phenotypes permutations.

Different Q-value cutoffs are represented in (a). Similar analysis with different after phenotype permutations (b). and different windows size in (c). The 3 local peaks identified at age 34, 60 and 78 are indicated by colored vertical lines. 
S8) DE-SWAN age effect when stratifying the analysis per cohort and accounting for subcohort in the modeling

a

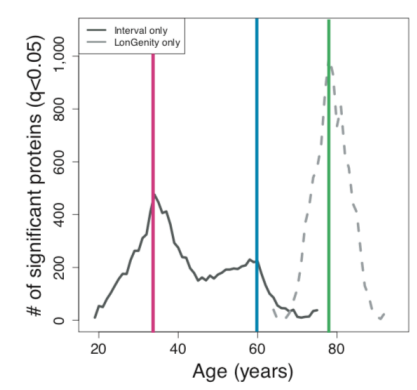

C

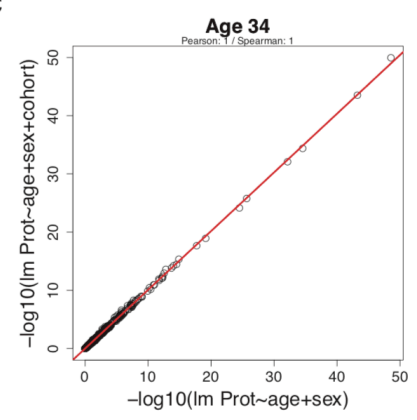

e

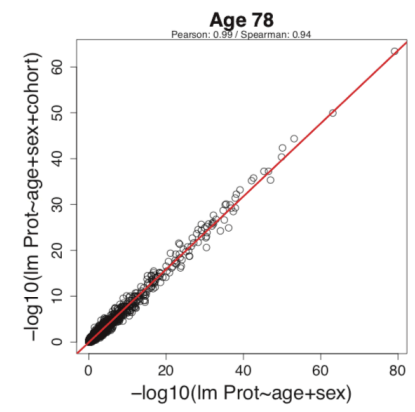

b

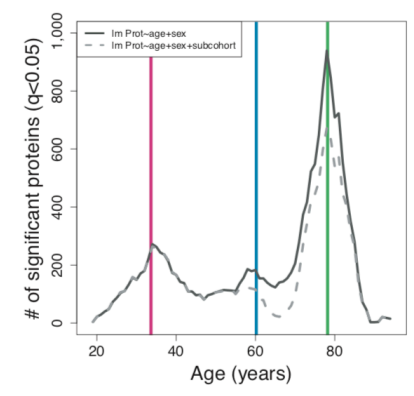

d

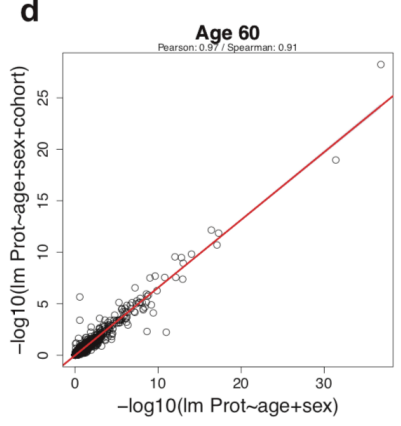

Supplementary Figure 8: DE-SWAN age effect when stratifying the analysis per cohort and accounting for subcohort.

(a) DE-SWAN results when stratifying the analysis per cohort. The 3 local peaks identified at age 34, 60 and 78y are indicated by colored vertical lines.

(b) Comparison of DE-SWAN results when adjusting for cohort or not. (c-e) Age effect at 34, 60 and 78y when adjusting for cohort or not. 
S9) Cis-associations and aging waves

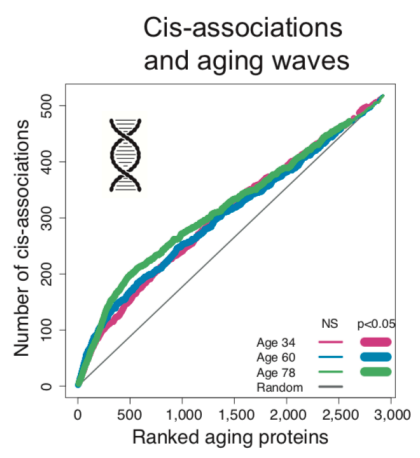

\section{Supplementary Figure 9: Cis-associations and aging waves.}

Enrichment for cis-association in the waves of aging proteins identified by DE-SWAN. Aging proteins were ranked based on p-values at age 34, 60 and 78 and the cumulative number of cisassociations was counted. Permutation based tests $(1 \mathrm{e}+5)$ were used to assess significance. 
S10) Age related changes of BMI.

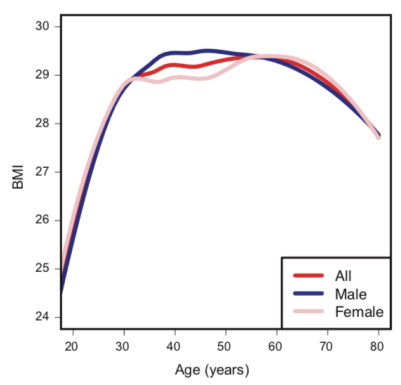

Supplementary Figure 10: Age related changes of BMI.

Changes during aging are estimated using LOESS curves, fitted for all subjects and for male and female separately. Data from the R NANHES package. 
S11) Normalization examples
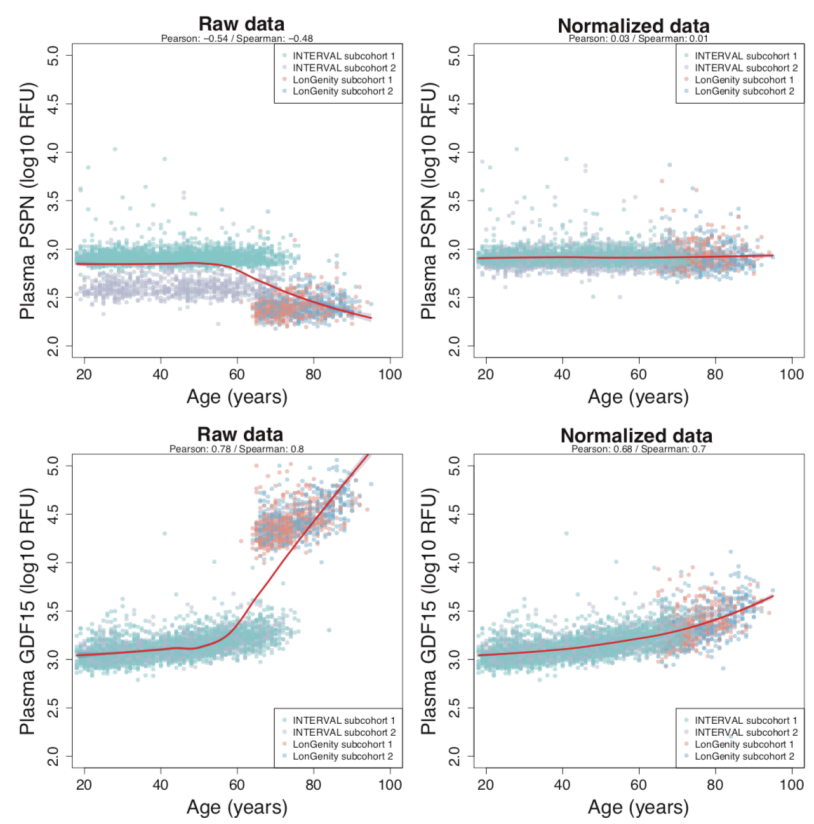

\section{Supplementary Figure 11: Normalization examples.}

Representative examples of the normalization process in the Interval and LonGenity cohorts. For each protein, the average of the subjects in the 60-70y range within each subcohort was used as a normalization factor. LOESS regression curves are fitted. Note that this normalization is needed when fitting aging trajectories (Fig. 2) but does not affect the results when "subcohort" is included as covariate in the statistical modeling. 
S12) Pathways covered by the proteins measured in this study

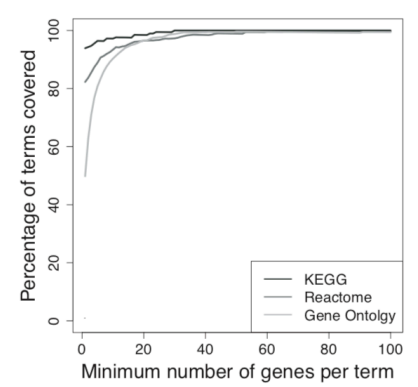

Supplementary Figure 12: Pathways covered by the 2,925 proteins measured in this study. The 2,925 proteins measured in this study cover $90 \%$ of the human GO, Reactome and KEGG terms containing more than 8 genes. Coverage for pathways with $1+$ to $100+$ genes is represented. 


\section{References}

1. Harman, D. The aging process: major risk factor for disease and death. Proc Natl Acad Sci U S A 88, 5360-5363 (1991).

2. Valdes, A.M., Glass, D. \& Spector, T.D. Omics technologies and the study of human ageing. Nat Rev Genet 14, 601-607 (2013).

3. Stegeman, R. \& Weake, V.M. Transcriptional Signatures of Aging. J Mol Biol 429, 24272437 (2017).

4. Aramillo Irizar, P., et al. Transcriptomic alterations during ageing reflect the shift from cancer to degenerative diseases in the elderly. Nat Commun 9, 327 (2018).

5. Baht, G.S., et al. Exposure to a youthful circulaton rejuvenates bone repair through modulation of beta-catenin. Nat Commun 6, 7131 (2015).

6. Conboy, I.M., et al. Rejuvenation of aged progenitor cells by exposure to a young systemic environment. Nature 433, 760-764 (2005).

7. Huang, Q., et al. A Young Blood Environment Decreases Aging of Senile Mice Kidneys. J Gerontol A Biol Sci Med Sci 73, 421-428 (2018).

8. Katsimpardi, L., et al. Vascular and neurogenic rejuvenation of the aging mouse brain by young systemic factors. Science 344, 630-634 (2014).

9. Loffredo, F.S., et al. Growth differentiation factor 11 is a circulating factor that reverses age-related cardiac hypertrophy. Cell 153, 828-839 (2013).

10. Salpeter, S.J., et al. Systemic regulation of the age-related decline of pancreatic beta-cell replication. Diabetes 62, 2843-2848 (2013).

11. Sinha, M., et al. Restoring systemic GDF11 levels reverses age-related dysfunction in mouse skeletal muscle. Science 344, 649-652 (2014).

12. Villeda, S.A., et al. The ageing systemic milieu negatively regulates neurogenesis and cognitive function. Nature 477, 90-94 (2011).

13. Villeda, S.A., et al. Young blood reverses age-related impairments in cognitive function and synaptic plasticity in mice. Nat Med 20, 659-663 (2014).

14. Castellano, J.M., et al. Human umbilical cord plasma proteins revitalize hippocampal function in aged mice. Nature 544, 488-492 (2017).

15. Zhou, J. \& Rossi, J. Aptamers as targeted therapeutics: current potential and challenges. Nat Rev Drug Discov 16, 440 (2017).

16. Emilsson, V., et al. Co-regulatory networks of human serum proteins link genetics to disease. Science 361, 769-773 (2018).

17. Gold, L., et al. Aptamer-based multiplexed proteomic technology for biomarker discovery. PloS one 5, e15004 (2010).

18. Austad, S.N. \& Fischer, K.E. Sex Differences in Lifespan. Cell metabolism 23, 10221033 (2016).

19. Ostan, R., et al. Gender, aging and longevity in humans: an update of an intriguing/neglected scenario paving the way to a gender-specific medicine. Clin Sci (Lond) 130, 1711-1725 (2016).

20. Tanaka, T., et al. Plasma proteomic signature of age in healthy humans. Aging Cell 17, e12799 (2018). 
21. Cohen, A.A. Aging across the tree of life: The importance of a comparative perspective for the use of animal models in aging. Biochimica et biophysica acta. Molecular basis of disease 1864, 2680-2689 (2018).

22. Somalogic. Non-human samples in the SomaScan ${ }^{\mathrm{TM}}$ assay. Technical Note. (2015).

23. Guiraud, S., et al. Identification of serum protein biomarkers for utrophin based DMD therapy. Scientific reports 7, 43697-43697 (2017).

24. Wang, R.N., et al. Bone Morphogenetic Protein (BMP) signaling in development and human diseases. Genes Dis 1, 87-105 (2014).

25. Sun, B.B., et al. Genomic atlas of the human plasma proteome. Nature 558, 73-79 (2018).

26. Sattlecker, M., et al. Alzheimer's disease biomarker discovery using SomaScan multiplexed protein technology. Alzheimers Dement 10, 724-734 (2014).

27. Sullivan, K.D., et al. Trisomy 21 causes changes in the circulating proteome indicative of chronic autoinflammation. Sci Rep 7, 14818 (2017).

28. Ganz, P., et al. Development and Validation of a Protein-Based Risk Score for Cardiovascular Outcomes Among Patients With Stable Coronary Heart Disease. JAMA 315, 2532-2541 (2016).

29. Carayol, J., et al. Protein quantitative trait locus study in obesity during weight-loss identifies a leptin regulator. Nat Commun 8, 2084 (2017).

30. Pruim, R. NHANES: Data from the US National Health and Nutrition Examination Study. (2015).

31. Go, A.S., et al. Heart disease and stroke statistics--2013 update: a report from the American Heart Association. Circulation 127, e6-e245 (2013).

32. Franceschi, C., Garagnani, P., Parini, P., Giuliani, C. \& Santoro, A. Inflammaging: a new immune-metabolic viewpoint for age-related diseases. Nat Rev Endocrinol 14, 576-590 (2018).

33. Franceschi, C., et al. The Continuum of Aging and Age-Related Diseases: Common Mechanisms but Different Rates. Front Med (Lausanne) 5, 61 (2018).

34. Horvath, S. \& Raj, K. DNA methylation-based biomarkers and the epigenetic clock theory of ageing. Nat Rev Genet 19, 371-384 (2018).

35. Castellano, J.M., Kirby, E.D. \& Wyss-Coray, T. Blood-Borne Revitalization of the Aged Brain. JAMA neurology 72, 1191-1194 (2015).

36. Wiklund, F.E., et al. Macrophage inhibitory cytokine-1 (MIC-1/GDF15): a new marker of all-cause mortality. Aging Cell 9, 1057-1064 (2010).

37. Cohen, E. \& Dillin, A. The insulin paradox: aging, proteotoxicity and neurodegeneration. Nat Rev Neurosci 9, 759-767 (2008).

38. Suhre, K., et al. Connecting genetic risk to disease end points through the human blood plasma proteome. Nat Commun 8, 14357 (2017).

39. Sha, S.J., et al. Safety, Tolerability, and Feasibility of Young Plasma Infusion in the Plasma for Alzheimer Symptom Amelioration Study: A Randomized Clinical Trial. JAMA neurology (2018).

40. Mehan, M.R., et al. Protein signature of lung cancer tissues. PloS one 7, e35157 (2012).

41. Di Angelantonio, E., et al. Efficiency and safety of varying the frequency of whole blood donation (INTERVAL): a randomised trial of 45000 donors. Lancet 390, 2360-2371 (2017). 
42. Gubbi, S., et al. Effect of Exceptional Parental Longevity and Lifestyle Factors on Prevalence of Cardiovascular Disease in Offspring. Am J Cardiol 120, 2170-2175 (2017).

43. Fox, J. \& Weisberg, S. An R Companion to Applied Regression, (SAGE Publications, 2011).

44. Benjamini, Y. \& Hochberg, Y. Controlling the False Discovery Rate: A Practical and Powerful Approach to Multiple Testing. Journal of the Royal Statistical Society. Series B (Methodological) 57, 289-300 (1995).

45. Ashburner, M., et al. Gene ontology: tool for the unification of biology. The Gene Ontology Consortium. Nat Genet 25, 25-29 (2000).

46. Kanehisa, M., Furumichi, M., Tanabe, M., Sato, Y. \& Morishima, K. KEGG: new perspectives on genomes, pathways, diseases and drugs. Nucleic Acids Res 45, D353D361 (2017).

47. Croft, D., et al. The Reactome pathway knowledgebase. Nucleic Acids Res 42, D472-477 (2014).

48. Alexa, A. \& Rahnenfuhrer, J. topGO: Enrichment Analysis for Gene Ontology. (2016).

49. Yu, G., Wang, L.G., Han, Y. \& He, Q.Y. clusterProfiler: an R package for comparing biological themes among gene clusters. OMICS 16, 284-287 (2012).

50. Carlson, M. org.Hs.eg.db: Genome wide annotation for Human. (2017).

51. Subramanian, A., et al. Gene set enrichment analysis: a knowledge-based approach for interpreting genome-wide expression profiles. Proc Natl Acad Sci U S A 102, 1554515550 (2005).

52. Castellano, J.M., et al. In vivo assessment of behavioral recovery and circulatory exchange in the peritoneal parabiosis model. Sci Rep 6, 29015 (2016).

53. Pagès, H., Aboyoun, P., Gentleman, R. \& DebRoy, S. Biostrings: Efficient manipulation of biological strings. (2019).

54. Dray, S. \& Dufour, A.B. The ade4 package: implementing the duality diagram for ecologists. Journal of Statistical Software 22, 1-20 (2007).

55. Friedman, J., Hastie, T. \& Tibshirani, R. Regularization Paths for Generalized Linear Models via Coordinate Descent. J Stat Softw 33, 1-22 (2010).

56. Lehallier, B., et al. Combined Plasma and Cerebrospinal Fluid Signature for the Prediction of Midterm Progression From Mild Cognitive Impairment to Alzheimer Disease. JAMA neurology 73, 203-212 (2016).

57. Wang, M., Zhao, Y. \& Zhang, B. Efficient Test and Visualization of Multi-Set Intersections. Sci Rep 5, 16923 (2015).

58. Epskamp, S., Cramer, A., Waldorp, L., Schmittmann, V. \& Borsboom, D. qgraph: Network Visualizations of Relationships in Psychometric Data. Journal of Statistical Software, Articles 48, 1-18 (2012). 